\title{
Null-Field Approach for Laplace Problems with Circular Boundaries Using Degenerate Kernels
}

\author{
Jeng-Tzong Chen, Wen-Cheng Shen \\ Department of Harbor and River Engineering, National Taiwan Ocean University, \\ Keelung 20224, Taiwan
}

Received 26 May 2006; accepted 12 December 2007

Published online 18 March 2008 in Wiley InterScience (www.interscience.wiley.com). DOI 10.1002/num.20332

\begin{abstract}
In this article, a semi-analytical method for solving the Laplace problems with circular boundaries using the null-field integral equation is proposed. The main gain of using the degenerate kernels is to avoid calculating the principal values. To fully utilize the geometry of circular boundary, degenerate kernels for the fundamental solution and Fourier series for boundary densities are incorporated into the null-field integral equation. An adaptive observer system is considered to fully employ the property of degenerate kernels in the polar coordinates. A linear algebraic system is obtained without boundary discretization. By matching the boundary condition, the unknown coefficients can be determined. The present method can be seen as one kind of semianalytical approaches since error only attributes to the truncated Fourier series. For the eccentric case, vector decomposition technique for the normal and tangential directions is carefully considered in implementing the hypersingular equation in mathematical essence although we transform it to summability to divergent series. The five advantages, well-posed linear algebraic system, principal value free, elimination of boundary-layer effect, exponential convergence, and mesh free, are achieved. Several examples involving infinite, half-plane, and bounded domains with circular boundaries are given to demonstrate the validity of the proposed method. (C) 2008 Wiley Periodicals, Inc. Numer Methods Partial Differential Eq 25: 63-86, 2009
\end{abstract}

Keywords: degenerate kernel; Fourier series; multiply-connected domain problem; null-field integral equation; semi-analytical approach

\section{INTRODUCTION}

The Laplace equation arises in many branches of physics, from which it recruits a wide group of researchers. Temperature in case of steady state heat conduction [1-6], electrostatic potential [7-11], velocity potential in a steady flow of an ideal fluid [12-14], the displacement of an infinite medium under remote uniformly shear [15-19], and the pure torsion of an elastic bar by equilibrated end torques $[20,21]$ are examples in which the Laplace equation is satisfied. Circular geometries often appear in engineering structures. Although these structures are very simple, the analytical solutions involve special mapping technique or restricted solution representations. Bipolar coordinates was always used to derive the analytical solution for two-holes 
problems [22]. However, conformal mapping or bipolar coordinates is limited to doubly connected regions; most efforts have concentrated on special solution representations. Alternative method has been adopted to solve problems with multiple circular holes [23,24]. Numerical approaches, e.g. finite difference method (FDM), finite element method (FEM), boundary element method (BEM), and meshfree method, etc. have been used to deal with engineering problems. Among diverse numerical approaches, FEM and BEM have become the popular research tools for engineers. In the past decades, FEM has been widely applied to solve many engineering problems, but one deficiency is that discretizations cost much time to set up the mesh models. Unlike FEM, the discretizations are restricted only to the boundary when using BEM. The main advantage of BEM is one-dimension reduction in mesh generation. For stress concentration problems, BEM can capture the local behavior. It is also ideally suited to the analysis of external problems where domains extend to infinity, since discretizations are confined to the internal boundaries with no need to truncate the domain at a finite distance. There is no doubt that BEM has been appreciated as an alternative numerical method which has been extensively used. Practical engineers and academic researchers paid attention to theoretical study and applications of BEM in the recent decades. Although BEM is recognized as an acceptable tool, some pitfalls still exist, e.g. degenerate scale for potential problems and fictitious frequency for exterior acoustics. Detailed discussions for the pitfalls of BEM can consult with the plenary lecture by Chen et al. [25].

For problems with circular boundaries, the BIEM can be utilized instead of BEM to improve the convergence by introducing Fourier series. The Fourier series expansion is specially tailored to problems with circular geometry. Early attempts to solve problems involving circular boundary using the series expansion were reported by Mogilevskaya et al. [26, 27], Barone and Caulk [2, 28-30], and Bird and Steele [3,31]. Barone and Caulk explored the use of special boundary integral method for solving Laplace's equation in two-dimensional regions with circular holes. On the basis of their idea, the boundary potential and its normal derivative were expressed in a finite series of circular harmonics on each hole. Unlike other approaches, the unknown coefficients in each hole are determined by a new set of integral equations with special kernel functions. However, the explicit equations in [20] were limited to the case when a constant potential is specified on the boundary of each hole. Bird and Steele $[3,31]$ have adopted the Fourier series for harmonic and biharmonic problems with circular holes. In their numerical results, only six terms of Fourier series on each hole were sufficient to yield error of less than 0.05 percent. More recently, Mogilevskaya and Crouch presented a method for solving problems with randomly distributed circular elastic inclusions with arbitrary properties [27]. They combined the series expansion technique with a direct boundary integral method. However, all of them didn't employ the null-field integral equation and degenerate kernels [32] in the polar coordinates to fully capture the circular boundary to the author's best knowledge although they have employed the Fourier series. The exponential convergence rate was proved in the Kress' book [32] for BIEM by using degenerate kernel and Fourier series. Numerical experiments was performed for the Stokes flow [33].

In the article, we focus on the problems with circular boundaries and possess a semianalytical approach. The comparison between the present approach and conventional BEM is arranged in Table I. A general-purpose program for circular boundaries with different radii and various positions of center is developed. A major benefit of using circular boundaries is that all integrations can be performed analytically. We expand the boundary density along the boundaries of each hole by using Fourier series expansion and employ the null-field integral equation to develop a system of linear algebraic equations. Several examples including multiple circular cavities are demonstrated to check the validity of the present method. Besides, half-plane problem with a circular hole is considered as the special case. 

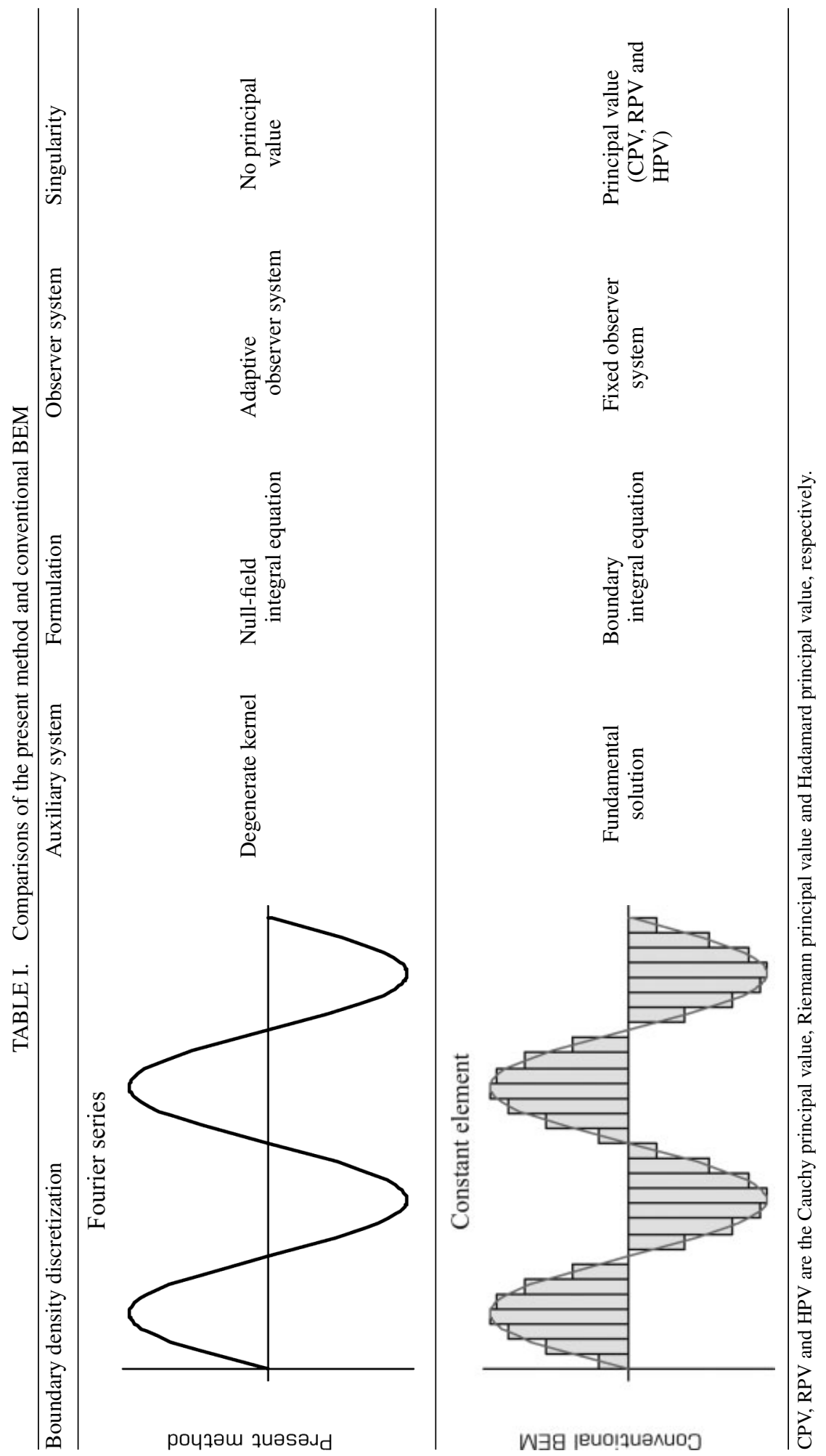


\section{PROBLEM STATEMENT AND MATHEMATICAL FORMULATION}

Suppose there are $N$ randomly distributed circular cavities bounded in the domain $D$ and enclosed with the boundary, $B_{k}(k=0,1,2, \ldots, N)$ as shown in Fig. 1 . We define

$$
B=\bigcup_{k=0}^{N} B_{k} .
$$

In mathematical physics, many engineering problems can be described by the Laplace equation, the governing equation is written below:

$$
\nabla^{2} u(\mathrm{x})=0, \quad \mathrm{x} \in D,
$$

where $\nabla^{2}$ is the Laplacian operator, $u(\mathrm{x})$ is the potential function and $D$ is the domain of interest. The integral equation for the domain point can be derived from the third Green's identity [34], we have

$$
\begin{aligned}
2 \pi u(\mathrm{x}) & =\int_{B} T(\mathrm{~s}, \mathrm{x}) u(\mathrm{~s}) d B(\mathrm{~s})-\int_{B} U(\mathrm{~s}, \mathrm{x}) t(\mathrm{~s}) d B(\mathrm{~s}), \mathrm{x} \in D, \\
2 \pi \frac{\partial u(\mathrm{x})}{\partial \mathbf{n}_{\mathrm{x}}} & =\int_{B} M(\mathrm{~s}, \mathrm{x}) u(\mathrm{~s}) d B(\mathrm{~s})-\int_{B} L(\mathrm{~s}, \mathrm{x}) t(\mathrm{~s}) d B(\mathrm{~s}), \mathrm{x} \in D,
\end{aligned}
$$

where $\mathrm{s}$ and $\mathrm{x}$ are the source and field points, respectively, $B$ is the boundary, $\mathbf{n}_{\mathrm{x}}$ denotes the outward normal vector at field point $\mathrm{x}$ (artificially defined, $(1,0)$ is for the $\mathrm{x}$ gradient and $(0,1)$ for the $y$ gradient on the interior point), and the kernel function

$U(\mathrm{~s}, \mathrm{x})=\ln r,(r \equiv|\mathrm{x}-\mathrm{s}|)$, is the fundamental solution which satisfies

$$
\nabla^{2} U(\mathrm{~s}, \mathrm{x})=2 \pi \delta(\mathrm{x}-\mathrm{s}),
$$

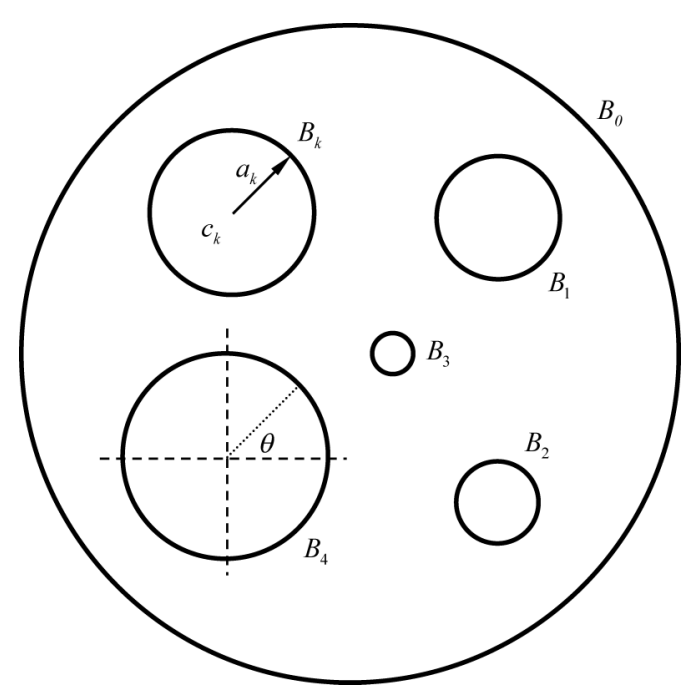

FIG. 1. Problem statements. 
in which $\delta(\mathrm{x}-\mathrm{s})$ denotes the Dirac-delta function. The other kernel functions, $T(\mathrm{~s}, \mathrm{x}), L(\mathrm{~s}, \mathrm{x})$ and $M(\mathrm{~s}, \mathrm{x})$, are defined by

$$
T(\mathrm{~s}, \mathrm{x}) \equiv \frac{\partial U(\mathrm{~s}, \mathrm{x})}{\partial \mathbf{n}_{\mathrm{s}}}, L(\mathrm{~s}, \mathrm{x}) \equiv \frac{\partial U(\mathrm{~s}, \mathrm{x})}{\partial \mathbf{n}_{\mathrm{x}}}, M(\mathrm{~s}, \mathrm{x}) \equiv \frac{\partial^{2} U(\mathrm{~s}, \mathrm{x})}{\partial \mathbf{n}_{\mathrm{s}} \partial \mathbf{n}_{\mathrm{x}}},
$$

where $\mathbf{n}_{\mathrm{s}}$ is the outward normal vector at the source point $\mathrm{s}$. By moving the field point to the boundary, the Eqs. (3) and (4) reduce to

$$
\begin{aligned}
\pi u(\mathrm{x}) & =\text { C.P.V. } \int_{B} T(\mathrm{~s}, \mathrm{x}) u(\mathrm{~s}) d B(\mathrm{~s})-\mathrm{R} . \mathrm{P} . \mathrm{V} \int_{B} U(\mathrm{~s}, \mathrm{x}) t(\mathrm{~s}) d B(\mathrm{~s}), \mathrm{x} \in B, \\
\pi \frac{\partial u(\mathrm{x})}{\partial \mathbf{n}_{\mathrm{x}}} & =\text { H.P.V. } \int_{B} M(\mathrm{~s}, \mathrm{x}) u(\mathrm{~s}) d B(\mathrm{~s})-\mathrm{C} . \mathrm{P} . \mathrm{V} \int_{B} L(\mathrm{~s}, \mathrm{x}) t(\mathrm{~s}) d B(\mathrm{~s}), \mathrm{x} \in B,
\end{aligned}
$$

where C.P.V., R.P.V., and H.P.V. denote the Cauchy principal value, Riemann principal value, and Hadamard principal value, respectively. Although $U, T, L$, and $M$ kernels are singular, we can deal with the singular integrals free of principal value sense due to the introduction of degenerate kernel. Once the field point $x$ locates outside the domain, the null-field integral equation of the direct method in Eqs. (7) and (8) yield

$$
\begin{aligned}
& 0=\int_{B} T(\mathrm{~s}, \mathrm{x}) u(\mathrm{~s}) d B(\mathrm{~s})-\int_{B} U(\mathrm{~s}, \mathrm{x}) t(\mathrm{~s}) d B(\mathrm{~s}), x \in D^{c} \cup B, \\
& 0=\int_{B} M(\mathrm{~s}, \mathrm{x}) u(\mathrm{~s}) d B(\mathrm{~s})-\int_{B} L(\mathrm{~s}, \mathrm{x}) t(\mathrm{~s}) d B(\mathrm{~s}), x \in D^{c} \cup B,
\end{aligned}
$$

where $D^{c}$ is the complementary domain. Note that the null-field integral equations are not singular since $s$ and $x$ never coincide. Equations (9) and (10) can include the boundary point collocation once the appropriate degenerate kernels are employed. The discontinuity of potentials due to $T$ and $L$ kernels automatically appears once the different degenerate expressions for $T$ and $L$ kernels are employed for the collocation point inside or outside the circular boundary. Readers can refer to the Appendix of [35].

\section{EXPANSIONS OF FUNDAMENTAL SOLUTION AND BOUNDARY DENSITY}

Now, we adopt the mathematical tools, degenerate kernels, and Fourier series for the purpose of analytical study. The combination of degenerate kernels and Fourier series plays the major role in handling problems with circular boundaries.

\section{Degenerate Kernels for Fundamental Solutions}

On the basis of the separable property, the kernel function $U(\mathrm{~s}, \mathrm{x})$ can be expanded into separable form by separating the source point and field point in the polar coordinates [36]:

$$
U(\mathrm{~s}, \mathrm{x})=\left\{\begin{array}{c}
U^{i}(R, \theta ; \rho, \phi)=\ln R-\sum_{m=1}^{\infty} \frac{1}{m}\left(\frac{\rho}{R}\right)^{m} \cos m(\theta-\phi), R \geq \rho \\
U^{e}(R, \theta ; \rho, \phi)=\ln \rho-\sum_{m=1}^{\infty} \frac{1}{m}\left(\frac{R}{\rho}\right)^{m} \cos m(\theta-\phi), \rho>R
\end{array},\right.
$$

where the superscripts " $i$ " and " $e$ " denote the interior $(R \geq \rho)$ and exterior $(\rho>R)$ cases, respectively. The origin of the observer system for the degenerate kernel is $(0,0)$. Figure 2 shows 


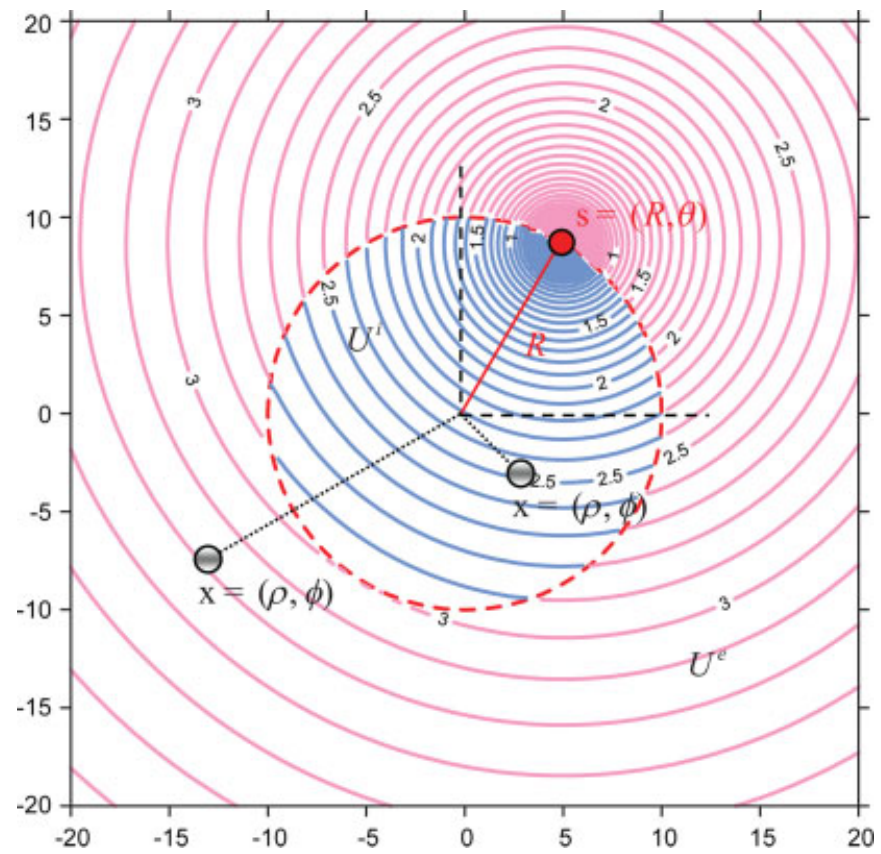

FIG. 2. Graph of the separable form of fundamental solution where the source s located at $R=10.0$ and $\theta=\frac{\pi}{3}$. [Color figure can be viewed in the online issue, which is available at www.interscience.wiley.com.]

the diagram of degenerate form for fundamental solution where the source point $\mathrm{s}$ located at $R=10.0, \theta=\pi / 3$. By setting the origin at $o$ for the observer system, a circle with a radius $R$ from the origin $o$ to the source point $\mathrm{s}$ is plotted. If the field point $\mathrm{x}$ is situated inside the circular region, the degenerate kernel belongs to the interior case $U^{i}$; otherwise, it is the exterior case. After taking the normal derivative with respect to Eq. (11), the $T(\mathrm{~s}, \mathrm{x})$ kernel can be derived as

$$
T(\mathrm{~s}, \mathrm{x})=\left\{\begin{array}{c}
T^{i}(R, \theta ; \rho, \phi)=\frac{1}{R}+\sum_{m=1}^{\infty}\left(\frac{\rho^{m}}{R^{m+1}}\right) \cos m(\theta-\phi), R>\rho \\
T^{e}(R, \theta ; \rho, \phi)=-\sum_{m=1}^{\infty}\left(\frac{R^{m-1}}{\rho^{m}}\right) \cos m(\theta-\phi), \rho>R
\end{array},\right.
$$

and the higher-order kernel functions, $L(\mathrm{~s}, \mathrm{x})$ and $M(\mathrm{~s}, \mathrm{x})$, are shown below

$$
\begin{gathered}
L(\mathrm{~s}, \mathrm{x})=\left\{\begin{array}{l}
L^{i}(R, \theta ; \rho, \phi)=-\sum_{m=1}^{\infty}\left(\frac{\rho^{m-1}}{R^{m}}\right) \cos m(\theta-\phi), R>\rho \\
L^{e}(R, \theta ; \rho, \phi)=\frac{1}{\rho}+\sum_{m=1}^{\infty}\left(\frac{R^{m}}{\rho^{m+1}}\right) \cos m(\theta-\phi), \rho>R
\end{array},\right. \\
M(\mathrm{~s}, \mathrm{x})=\left\{\begin{array}{l}
M^{i}(R, \theta ; \rho, \phi)=\sum_{m=1}^{\infty}\left(\frac{m \rho^{m-1}}{R^{m+1}}\right) \cos m(\theta-\phi), R \geq \rho \\
M^{e}(R, \theta ; \rho, \phi)=\sum_{m=1}^{\infty}\left(\frac{m R^{m-1}}{\rho^{m+1}}\right) \cos m(\theta-\phi), \rho>R
\end{array}\right.
\end{gathered}
$$

Since the potential resulted from $T(\mathrm{~s}, \mathrm{x})$ and $L(\mathrm{~s}, \mathrm{x})$ kernels are discontinuous across the boundary, the potentials of $T(\mathrm{~s}, \mathrm{x})$ for $R \rightarrow \rho^{+}$and $R \rightarrow \rho^{-}$are different. This is the reason why $R=\rho$ is not included in the expression of degenerate kernels for $T(\mathrm{~s}, \mathrm{x})$ and $L(\mathrm{~s}, \mathrm{x})$ in Eqs. (12) and (13). 


\section{Fourier Series Expansion for Unknown Boundary Densities}

We apply the Fourier series expansion to approximate the potential $u$ and its normal derivative on the boundary

$$
\begin{aligned}
& u\left(\mathrm{~s}_{k}\right)=a_{0}^{k}+\sum_{n=1}^{\infty}\left(a_{n}^{k} \cos n \theta_{k}+b_{n}^{k} \sin n \theta_{k}\right), \mathrm{s}_{k} \in B_{k}, k=1,2, \ldots, N, \\
& t\left(\mathrm{~s}_{k}\right)=p_{0}^{k}+\sum_{n=1}^{\infty}\left(p_{n}^{k} \cos n \theta_{k}+q_{n}^{k} \sin n \theta_{k}\right), \mathrm{s}_{k} \in B_{k}, k=1,2, \ldots, N,
\end{aligned}
$$

where $a_{n}^{k}, b_{n}^{k}, p_{n}^{k}$ and $q_{n}^{k}(n=0,1,2, \cdots)$ are the Fourier coefficients and $\theta_{k}$ is the polar angle measured related to the $x$-direction.

\section{MATHEMATICAL FORMULATION AND SOLUTION PROCEDURES}

\section{A. Adaptive Observer System}

In real implementation, the collocation point $\mathrm{x}$ in Eq. (9) can be exactly located on the boundary since the degenerate kernel is introduced. To analytically carry out the $i$ th circular integral in Eq. (9), we adaptively set the origin of the observer at the center of the $i$ th circle. Adaptive observer system is chosen to fully employ the property of degenerate kernels. Figure $3(a, b)$ show the boundary integration for the circular boundaries in the adaptive observer system. The key idea of the present approach is that we employ the null field formulation and we can collocate the point $\mathrm{x}$ on the real boundary due to the introduction of degenerate kernel. It is worthy of note that the origin of the observer system is located at the center of the corresponding integrating circular boundary to entirely utilize the geometry of circular boundary for the expansion of degenerate kernels and boundary densities. The dummy variable in the circular integration is the angle $(\theta)$ instead of the radial ordinate $(R)$.

\section{B. Linear Algebraic Equation}

By moving the null-field point $\mathrm{x}_{k}$ to the $k$ th circular boundary in the sense of limit for Eq. (9) in Fig. 3(a), we have

$$
0=\sum_{k=0}^{N} \int_{B_{k}} T\left(\mathrm{~s}_{k}, \mathrm{x}_{j}\right) u_{k}(\mathrm{~s}) d B_{k}(\mathrm{~s})-\sum_{k=0}^{N} \int_{B_{k}} U\left(\mathrm{~s}_{k}, \mathrm{x}_{j}\right) t_{k}(\mathrm{~s}) d B_{k}(\mathrm{~s}), \mathrm{x}_{j} \in D^{c} .
$$

In the real computation, we select the collocation point on the boundary. If the domain is unbounded, the outer boundary $B_{0}$ is a circle with an infinite radius. It is noted that the integration path is counterclockwise for the outer circle. Otherwise, it is clockwise. For the $B_{k}$ integral of the circular boundary, the kernels of $U(\mathrm{~s}, \mathrm{x})$ and $T(\mathrm{~s}, \mathrm{x})$ are, respectively, expressed in terms of degenerate kernels of Eqs. (11) and (12), and $u(\mathrm{~s})$ and $t(\mathrm{~s})$ are substituted by using the Fourier series of Eqs. (15) and (16), respectively. In the $B_{k}$ integration, we set the origin of the observer system at the center $c_{k}$ to fully utilize the degenerate kernels and Fourier series. By collocating the null-field point on the boundary from outside of the domain, a linear algebraic system is obtained

$$
[\mathbf{U}]\{\mathbf{t}\}=[\mathbf{T}]\{\mathbf{u}\},
$$




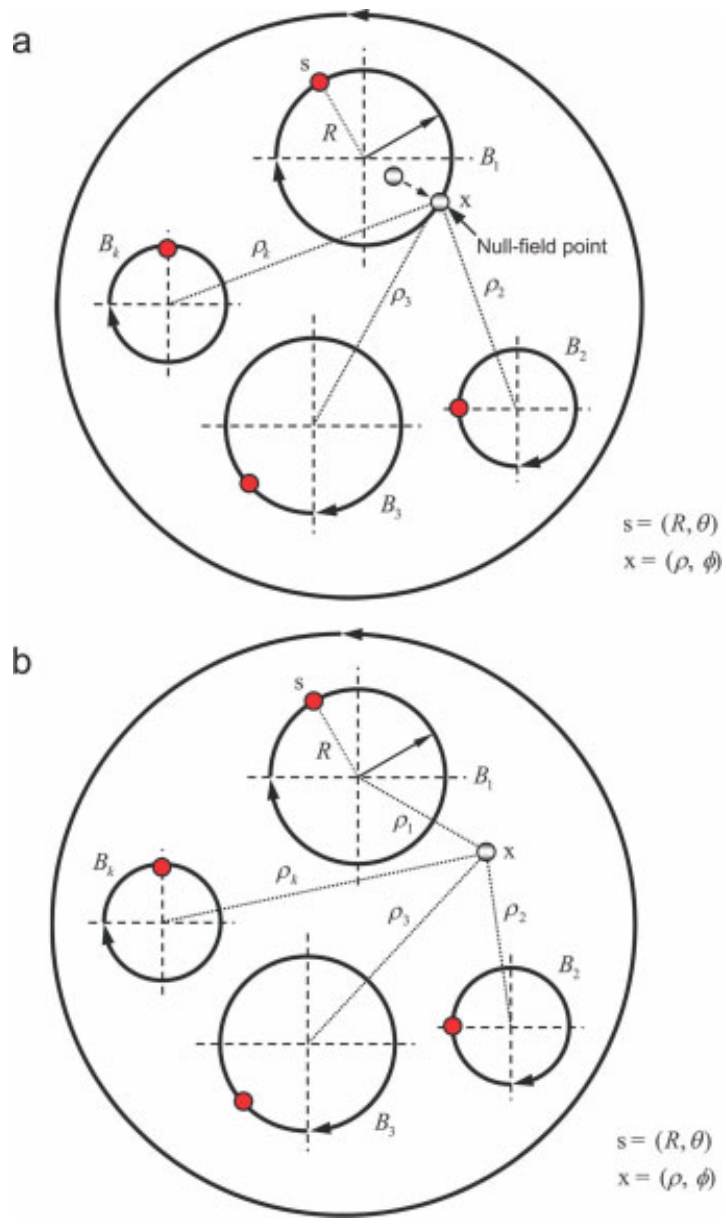

FIG. 3. (a) Sketch of the null-field integral equation in conjunction with the adaptive observer system, (b) sketch of the boundary integral equation for domain point in conjunction with the adaptive observer system. [Color figure can be viewed in the online issue, which is available at www.interscience.wiley.com.]

where $[\mathbf{U}]$ and $[\mathbf{T}]$ are the influence matrices with a dimension of $N(2 M+1)$ by $N(2 M+1)$, $\{\mathbf{u}\}$, and $\{\mathbf{t}\}$ denote the column vectors of Fourier coefficients with a dimension of $N(2 M+1)$ by 1 in which $M$ indicates the number of truncated terms in Fourier series, $[\mathbf{U}],[\mathbf{T}],\{\mathbf{u}\}$, and $\{\mathbf{t}\}$ can be defined as follows:

$$
\begin{gathered}
{[\mathbf{U}]=\left[\begin{array}{cccc}
\mathbf{U}_{00} & \mathbf{U}_{01} & \cdots & \mathbf{U}_{0 N} \\
\mathbf{U}_{10} & \mathbf{U}_{11} & \cdots & \mathbf{U}_{1 N} \\
\vdots & \vdots & \ddots & \vdots \\
\mathbf{U}_{N 0} & \mathbf{U}_{N 1} & \cdots & \mathbf{U}_{N N}
\end{array}\right],[\mathbf{T}]=\left[\begin{array}{cccc}
\mathbf{T}_{00} & \mathbf{T}_{01} & \cdots & \mathbf{T}_{0 N} \\
\mathbf{T}_{10} & \mathbf{T}_{11} & \cdots & \mathbf{T}_{1 N} \\
\vdots & \vdots & \ddots & \vdots \\
\mathbf{T}_{N 0} & \mathbf{T}_{N 1} & \cdots & \mathbf{T}_{N N}
\end{array}\right],} \\
\{\mathbf{u}\}=\left\{\begin{array}{c}
\mathbf{u}_{0} \\
\mathbf{u}_{1} \\
\mathbf{u}_{2} \\
\vdots \\
\mathbf{u}_{N}
\end{array}\right\},\{\mathbf{t}\}=\left\{\begin{array}{c}
\mathbf{t}_{0} \\
\mathbf{t}_{1} \\
\mathbf{t}_{2} \\
\vdots \\
\mathbf{t}_{N}
\end{array}\right\},
\end{gathered}
$$


where the vectors $\left\{\mathbf{u}_{k}\right\}$ and $\left\{\mathbf{t}_{k}\right\}$ are in the form of $\left\{a_{0}^{k} a_{1}^{k} b_{1}^{k} \cdots a_{M}^{k} b_{M}^{k}\right\}^{T}$ and $\left\{p_{0}^{k} p_{1}^{k} q_{1}^{k} \cdots p_{M}^{k} q_{M}^{k}\right\}^{T}$, respectively; the first subscript " $j$ " $(j=0,1,2, \ldots, N)$ in $\left[\mathbf{U}_{j k}\right]$ and $\left[\mathbf{T}_{j k}\right]$ denotes the index of the $j$ th circle where the collocation point is located and the second subscript " $k$ " $(k=0,1,2, \ldots, N)$ denotes the index of the $k$ th circle where boundary data $\left\{\mathbf{u}_{k}\right\}$ or $\left\{\mathbf{t}_{k}\right\}$ are specified, $N$ is the number of circular holes in the domain. The coefficient matrix of the linear algebraic system is partitioned into blocks, and each off-diagonal block corresponds to the influence matrices between two different circular cavities. The diagonal blocks are the influence matrices due to itself in each individual hole. After uniformly collocating the point along the $j$ th circular boundary, the submatrix can be written as

$$
\begin{aligned}
& {\left[\mathbf{U}_{j k}\right]=\left[\begin{array}{cccccc}
U_{j k}^{0 c}\left(\phi_{1}\right) & U_{j k}^{1 c}\left(\phi_{1}\right) & U_{j k}^{1 s}\left(\phi_{1}\right) & \cdots & U_{j k}^{M c}\left(\phi_{1}\right) & U_{j k}^{M s}\left(\phi_{1}\right) \\
U_{j k}^{0 c}\left(\phi_{2}\right) & U_{j k}^{1 c}\left(\phi_{2}\right) & U_{j k}^{1 s}\left(\phi_{2}\right) & \cdots & U_{j k}^{M c}\left(\phi_{2}\right) & U_{j k}^{M s}\left(\phi_{2}\right) \\
U_{j k}^{0 c}\left(\phi_{3}\right) & U_{j k}^{1 c}\left(\phi_{3}\right) & U_{j k}^{1 s}\left(\phi_{3}\right) & \cdots & U_{j k}^{M c}\left(\phi_{3}\right) & U_{j k}^{M s}\left(\phi_{3}\right) \\
\vdots & \vdots & \vdots & \ddots & \vdots & \vdots \\
U_{j k}^{0 c}\left(\phi_{2 M}\right) & U_{j k}^{1 c}\left(\phi_{2 M}\right) & U_{j k}^{1 s}\left(\phi_{2 M}\right) & \cdots & U_{j k}^{M c}\left(\phi_{2 M}\right) & U_{j k}^{M s}\left(\phi_{2 M}\right) \\
U_{j k}^{0 c}\left(\phi_{2 M+1}\right) & U_{j k}^{1 c}\left(\phi_{2 M+1}\right) & U_{j k}^{1 s}\left(\phi_{2 M+1}\right) & \cdots & U_{j k}^{M c}\left(\phi_{2 M+1}\right) & U_{j k}^{M s}\left(\phi_{2 M+1}\right)
\end{array}\right],} \\
& {\left[\mathbf{T}_{j k}\right]=\left[\begin{array}{cccccc}
T_{j k}^{0 c}\left(\phi_{1}\right) & T_{j k}^{1 c}\left(\phi_{1}\right) & T_{j k}^{1 s}\left(\phi_{1}\right) & \cdots & T_{j k}^{M c}\left(\phi_{1}\right) & T_{j k}^{M s}\left(\phi_{1}\right) \\
T_{j k}^{0 c}\left(\phi_{2}\right) & T_{j k}^{1 c}\left(\phi_{2}\right) & T_{j k}^{1 s}\left(\phi_{2}\right) & \cdots & T_{j k}^{M c}\left(\phi_{2}\right) & T_{j k}^{M s}\left(\phi_{2}\right) \\
T_{j k}^{0 c}\left(\phi_{3}\right) & T_{j k}^{1 c}\left(\phi_{3}\right) & T_{j k}^{1 s}\left(\phi_{3}\right) & \cdots & T_{j k}^{M c}\left(\phi_{3}\right) & T_{j k}^{M s}\left(\phi_{3}\right) \\
\vdots & \vdots & \vdots & \ddots & \vdots & \vdots \\
T_{j k}^{0 c}\left(\phi_{2 M}\right) & T_{j k}^{1 c}\left(\phi_{2 M}\right) & T_{j k}^{1 s}\left(\phi_{2 M}\right) & \cdots & T_{j k}^{M c}\left(\phi_{2 M}\right) & T_{j k}^{M s}\left(\phi_{2 M}\right) \\
T_{j k}^{0 c}\left(\phi_{2 M+1}\right) & T_{j k}^{1 c}\left(\phi_{2 M+1}\right) & T_{j k}^{1 s}\left(\phi_{2 M+1}\right) & \cdots & T_{j k}^{M c}\left(\phi_{2 M+1}\right) & T_{j k}^{M s}\left(\phi_{2 M+1}\right)
\end{array}\right] .}
\end{aligned}
$$

It is noted that the superscript " $0 s$ " in Eqs. (21) and (22) disappears since $\sin \theta=0$. The elements of $\left[\mathbf{U}_{j k}\right]$ and $\left[\mathbf{T}_{j k}\right]$ are defined respectively as

$$
\begin{aligned}
U_{j k}^{n c}\left(\phi_{m}\right) & =\int_{B_{k}} U\left(\mathrm{~s}_{k}, \mathrm{x}_{m}\right) \cos \left(n \theta_{k}\right) R_{k} d \theta_{k}, n=0,1,2, \ldots, M, m=1,2, \ldots, 2 M+1, \\
U_{j k}^{n s}\left(\phi_{m}\right) & =\int_{B_{k}} U\left(\mathrm{~s}_{k}, \mathrm{x}_{m}\right) \sin \left(n \theta_{k}\right) R_{k} d \theta_{k}, n=1,2, \ldots, M, m=1,2, \ldots, 2 M+1, \\
T_{j k}^{n s}\left(\phi_{m}\right) & =\int_{B_{k}} T\left(\mathrm{~s}_{k}, \mathrm{x}_{m}\right) \cos \left(n \theta_{k}\right) R_{k} d \theta_{k}, n=0,1,2, \ldots, M, m=1,2, \ldots, 2 M+1, \\
T_{j k}^{n s}\left(\phi_{m}\right) & =\int_{B_{k}} T\left(\mathrm{~s}_{k}, \mathrm{x}_{m}\right) \sin \left(n \theta_{k}\right) R_{k} d \theta_{k}, n=1,2, \ldots, M, m=1,2, \ldots, 2 M+1,
\end{aligned}
$$

where $\phi_{m}$ is the polar angle of the collocating points $\mathrm{x}_{m}$ along the boundary.

All the undetermined coefficients are easily determined by using Eq. (18) and the prescribed boundary conditions. Then the unknown boundary values can be determined and the potential at any point can be obtained according to Eq. (3). 


\section{Vector Decomposition Technique for the Potential Gradient in the Hyper-Singular Equation}

Equation (4) shows the normal derivative of potential for domain points, special treatment is considered here. Since the hypersingular equation in mathematical essence is also an alternative to deal with the problem of degenerate scale [37-39], potential gradient on the boundary is required to calculate. For the nonconcentric case, special treatment for the normal derivative should be taken care as the source point and field point locate on different circular boundaries. As shown in Fig. 4, the normal direction on the boundary $\left(1,1^{\prime}\right)$ should be superimposed by the radial derivative $\left(3,3^{\prime}\right)$ and angular derivative $\left(4,4^{\prime}\right)$. We called this treatment "vector decomposition technique." According to the concept of vector decomposition technique, Eqs. (13) and (14) can be modified as

$$
\begin{aligned}
& L(\mathrm{~s}, \mathrm{x})=\left\{\begin{array}{rl}
L^{i}(R, \theta ; \rho, \phi)= & -\sum_{m=1}^{\infty}\left(\frac{\rho^{m-1}}{R^{m}}\right) \cos m(\theta-\phi) \cos (\zeta-\xi) \\
& -\sum_{m=1}^{\infty}\left(\frac{\rho^{m-1}}{R^{m}}\right) \sin m(\theta-\phi) \cos \left(\frac{\pi}{2}-\zeta+\xi\right), R>\rho \\
L^{e}(R, \theta ; \rho, \phi)= & \frac{1}{\rho}+\sum_{m=1}^{\infty}\left(\frac{R^{m}}{\rho^{m+1}}\right) \cos m(\theta-\phi) \cos (\zeta-\xi) \\
& -\sum_{m=1}^{\infty}\left(\frac{R^{m}}{\rho^{m+1}}\right) \sin m(\theta-\phi) \cos \left(\frac{\pi}{2}-\zeta+\xi\right), \rho>R
\end{array},\right. \\
& M(\mathrm{~s}, \mathrm{x})=\left\{\begin{array}{rl}
M^{i}(R, \theta ; \rho, \phi)= & \sum_{m=1}^{\infty}\left(\frac{m \rho^{m-1}}{R^{m+1}}\right) \cos m(\theta-\phi) \cos (\zeta-\xi) \\
& -\sum_{m=1}^{\infty}\left(\frac{m \rho^{m-1}}{R^{m+1}}\right) \sin m(\theta-\phi) \cos \left(\frac{\pi}{2}-\zeta+\xi\right), R \geq \rho \\
M^{e}(R, \theta ; \rho, \phi)= & \sum_{m=1}^{\infty}\left(\frac{m R^{m-1}}{\rho^{m+1}}\right) \cos m(\theta-\phi) \cos (\zeta-\xi) \\
& -\sum_{m=1}^{\infty}\left(\frac{m R^{m-1}}{\rho^{m+1}}\right) \sin m(\theta-\phi) \cos \left(\frac{\pi}{2}-\zeta+\xi\right), \rho>R
\end{array},\right.
\end{aligned}
$$

where $\zeta$ and $\xi$ are shown in Fig. 4. For the special case, the circles with respect to the same origin of observer, the potential gradient is derived free of special treatment since $\zeta=\xi$.

\section{ILLUSTRATIVE EXAMPLES AND DISCUSSIONS}

Different branches of engineering applications are given to test our formulation, e.g. steady state heat conduction, electrostatic potential of wires, and flow of an ideal fluid past cylinders. We will introduce the three topics item by item as follows:

\section{A. Steady State Heat Conduction Problems}

One example derived by Carrier and Pearson [40] and three problems with different boundary conditions found in Caulk's article [4] are considered.

Case 1. Eccentric case. An eccentric case with radii $a_{1}$ and $a_{2}\left(a_{1}=2.5, a_{2}=1.0\right)$ is shown in Fig. 5(a). The boundary condition on the inner hole is $u=0$ and the potential on the outer circle 


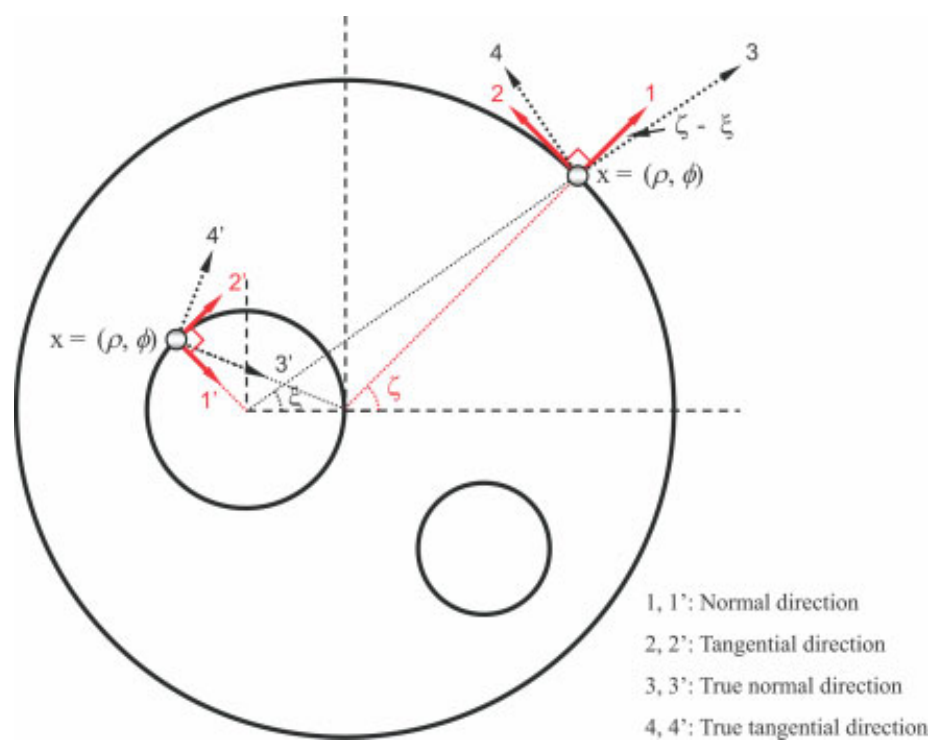

FIG. 4. Vector decomposition for the potential gradient in the hypersingular equation. [Color figure can be viewed in the online issue, which is available at www.interscience.wiley.com.]

is $u=1$. The numerical result is shown in Fig. 5(b). Good agreement is made after comparing the result with the exact solution [10],

$$
u(\rho, \phi)=\frac{1}{2 \ln 2} \ln \left[\frac{16 \rho^{2}+1+8 \rho \cos \phi}{\rho^{2}+16+8 \rho \cos \phi}\right],
$$

as shown in Fig. 5(c). After determining the Fourier coefficients, we obtain the normal flux on boundaries. The error distribution of flux on the inner boundary using different approaches is shown in Fig. 6. Since error only stems from the truncated terms of Fourier series using the present formulation, the results are better than those using BEM. In addition, the results of present method are also compared with Trefftz method and MFS (Method of Fundamental Solution). Furthermore, we adopted the Parseval's theorem to study the convergence rate with different number of terms in Fourier series since the boundary densities are continuous on $[0,2 \pi]$. The Parserval's theorem are defined as below

$$
\int_{0}^{2 \pi}[f(\theta)]^{2} d \theta \doteqdot 2 \pi a_{0}^{2}+\pi \sum_{n=1}^{M}\left(a_{n}^{2}+b_{n}^{2}\right) .
$$

According to Eq. (30), we have the Parseval's sum versus different number of terms in Fourier series for inner circle which are plotted in Fig. 7. Only a few terms of Fourier series are required to yield acceptable results.

Case 2. Two circular holes in a circle. A circular region of radius $R_{0}$ contains two circular holes which are placed at a distance $b(b=1)$ from the origin. Both holes have the same radius $a$ as shown in Fig. 8(a). The radii of the circular holes and the external boundary are $a=0.5$ and $R_{0}=2.0$. The results are shown in Fig. 8(b). After comparing the result 
a

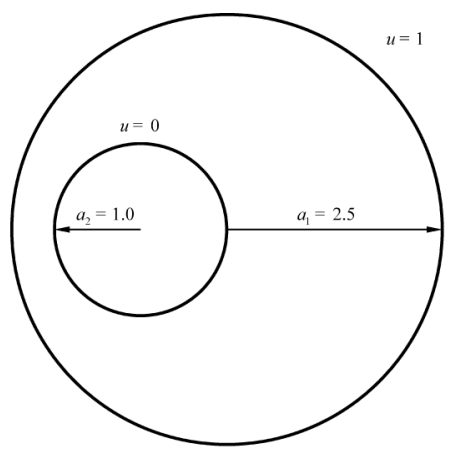

b

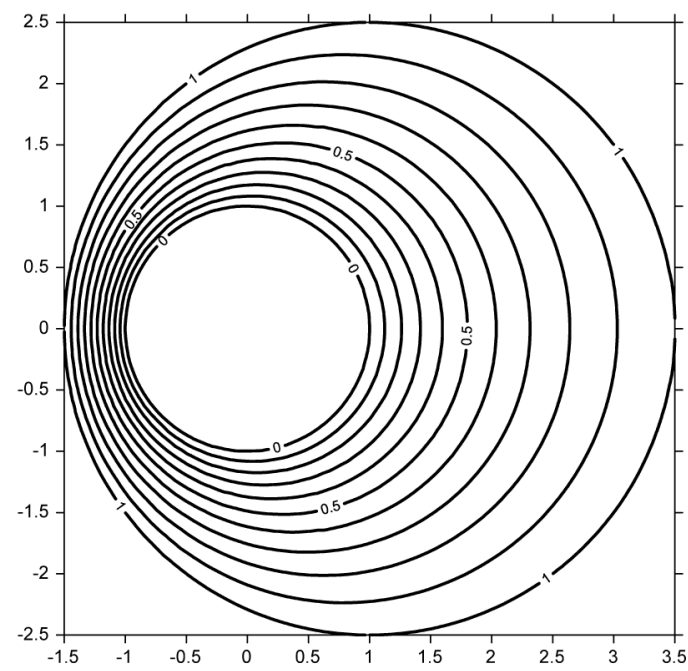

C

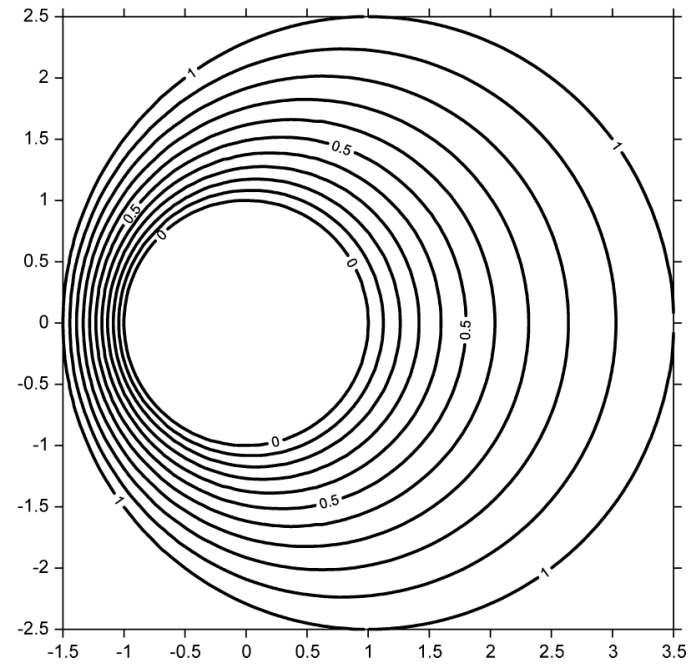

FIG. 5. (a) Laplace problem for the eccentric case, (b) contour plot for the present method (42 collocation points), (c) contour plot for the exact solution [40]. 


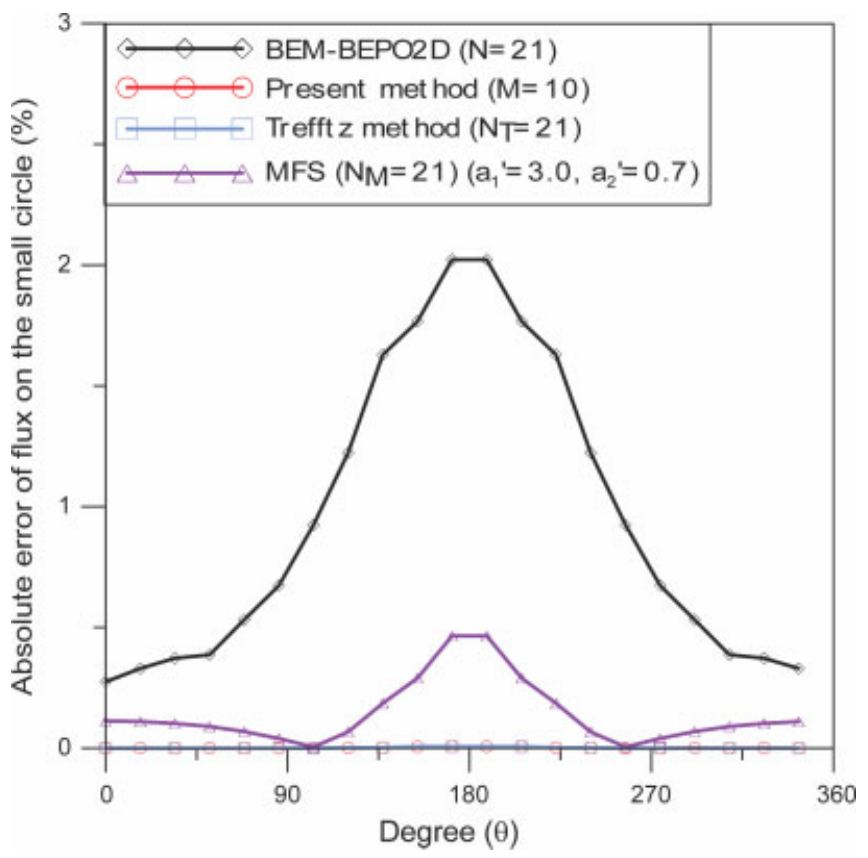

FIG. 6. The relative error distribution for eccentric case using four methods ( $a_{1}=2.5$, normal scale). [Color figure can be viewed in the online issue, which is available at www.interscience.wiley.com.]

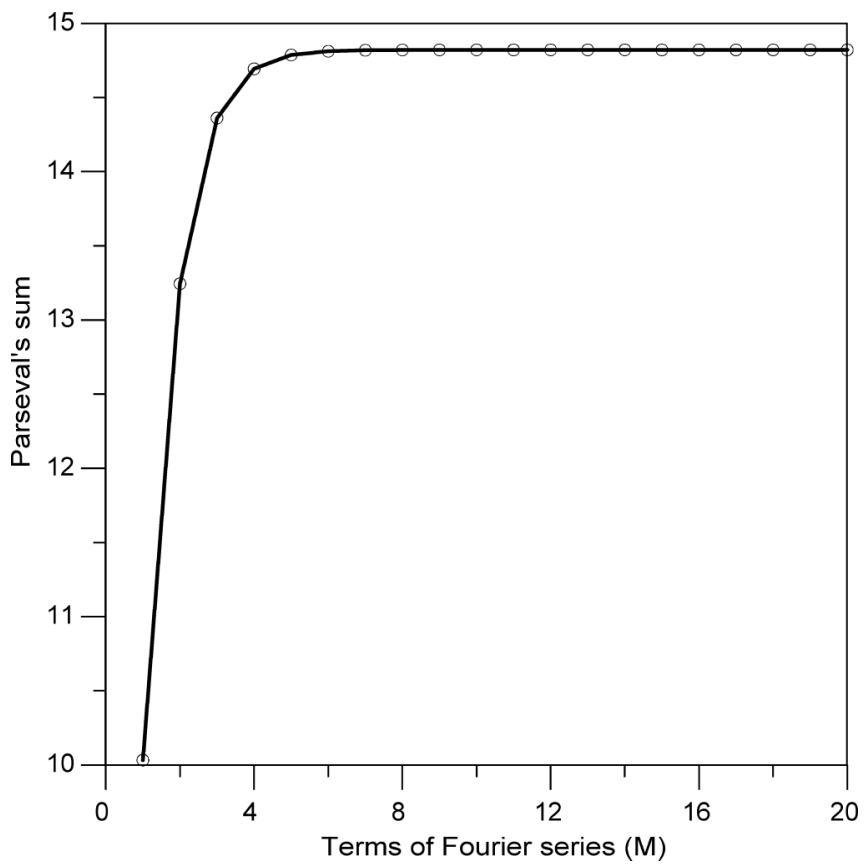

FIG. 7. Parseval's sum for the inner circle. 

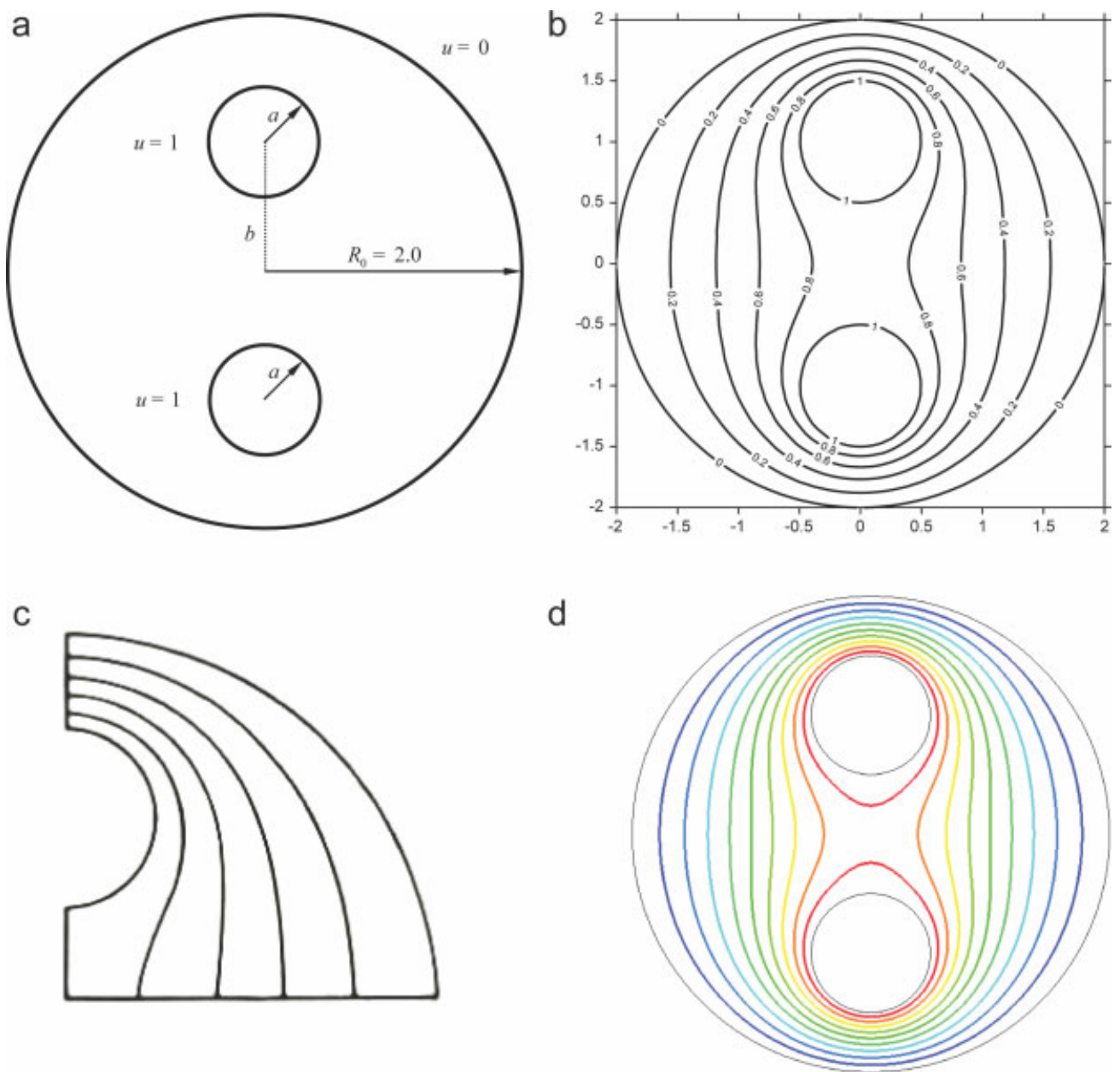

FIG. 8. (a) Problem statements, (b) contour plot for the present method (63 collocation points), (c) quarter part of contour plot for Caulk's data [4], (d) contour plot for FEM-ABAQUS (6502 elements). [Color figure can be viewed in the online issue, which is available at www.interscience.wiley.com.]

with the quarter part of Caulk's result in Fig. 8(c) [4], good agreement is made. ABAQUS shows the result as well as the present procedure with plenty of elements (6502 elements) in Fig. 8(d).

Case 3. Three circular holes in a circle. Consider the same region as in the above example, but now add a hole of radius $c$ at the center. The boundary conditions are different from each other as shown in Fig. 9(a). The radii of the holes are $a=c=0.4$ and the distance $b=1.2$ from the center of the external boundary. Figure 9(b) shows the numerical results obtained using the present method. The quarter part of the potential by Caulk [4] is shown in Fig. 9(c) for comparison, good agreement is made. Figure 9(d) shows the potential contour using ABAQUS. 
a

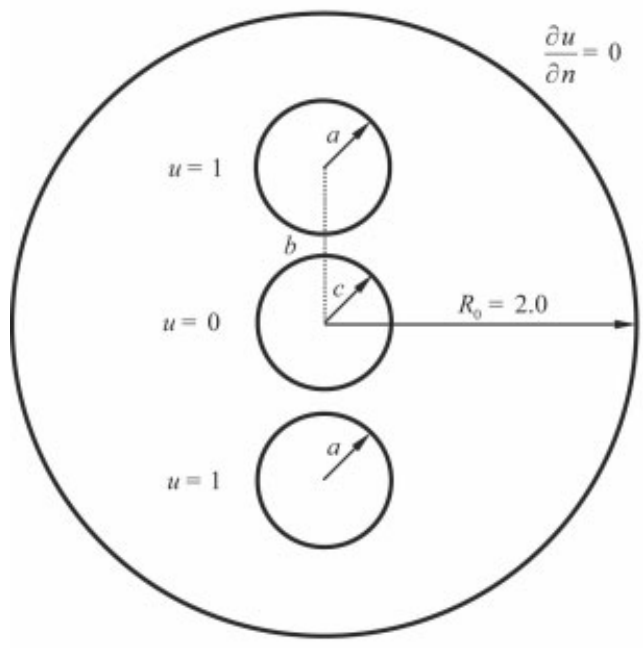

$\mathrm{C}$

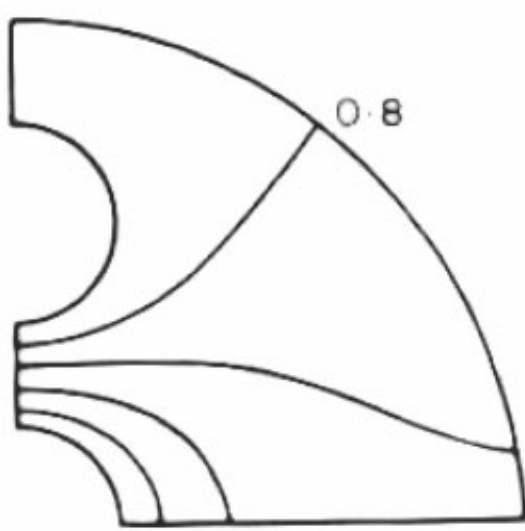

b

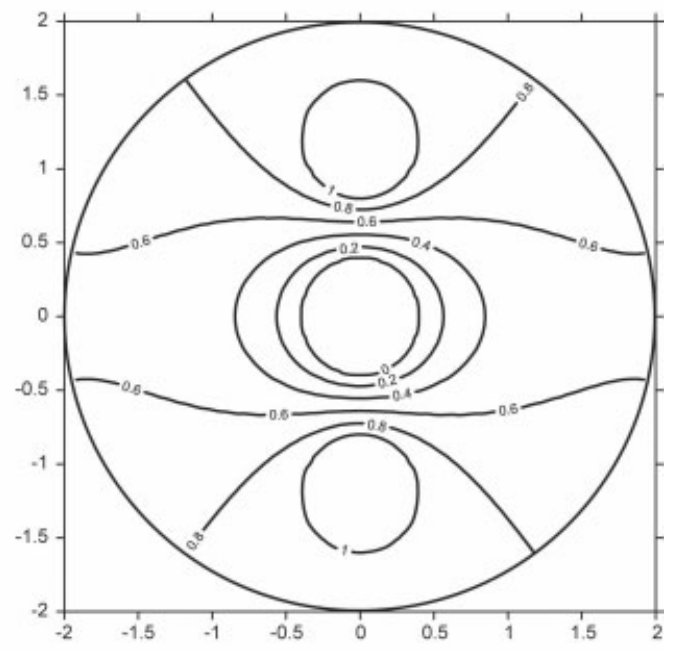

d

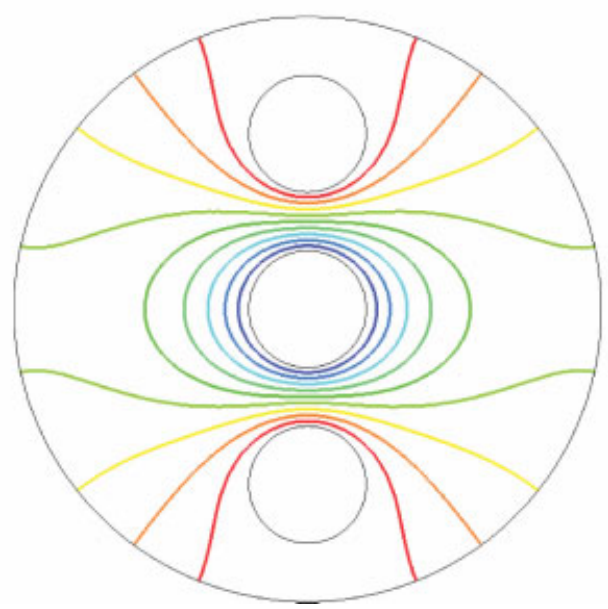

FIG. 9. (a) Problem statements, (b) contour plot for the present method (84 collocation points), (c) quarter part of contour plot for Caulk's data [4], (d) contour plot for FEM-ABAQUS (8050 elements). [Color figure can be viewed in the online issue, which is available at www.interscience.wiley.com.]

\section{B. Electrostatic Potential of Wires}

Case 1. Electrostatic potential of two parallel cylinders. The first electrostatic of wires problem reported in [22] is offered to verify the present procedure. Two circular cylindrical conductors of radius $a$ with centers at distance $2 l$ from each other are charged to potentials 1 and -1 , respectively (see Fig. 10(a)). Numerical results and exact solution by Lebedev et al. are shown in Fig. 10(b, c), respectively.

Case 2. Hexagonal electrostatic potential. The other one is a hexagonal electrostatic field of wires which has been solved by Onishi [41]. All the wires have equal radii and the boundary conditions are shown in Fig. 11(a). Figure 11(b) agrees well with Onishi's data as shown in Fig. 11(c). 
a
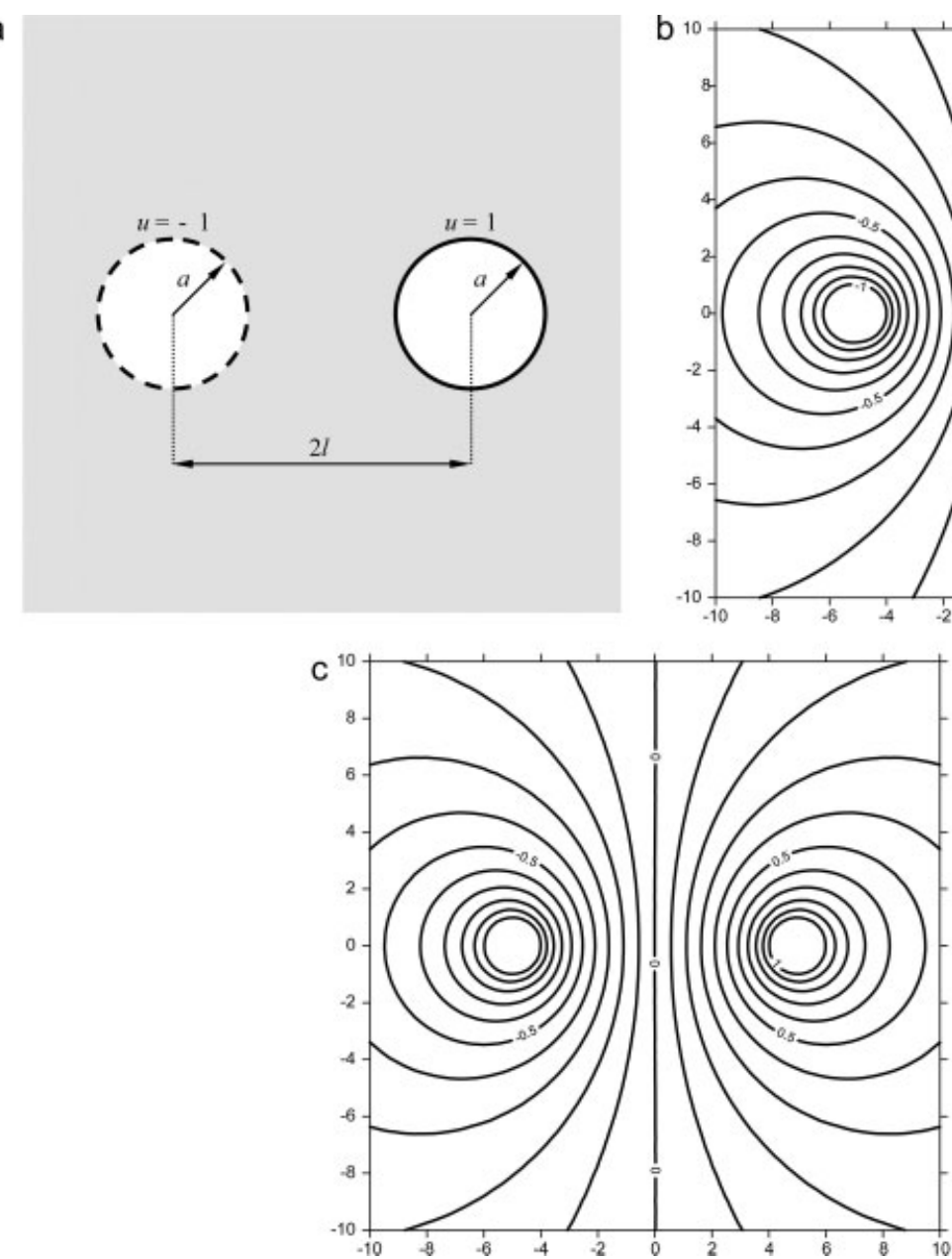

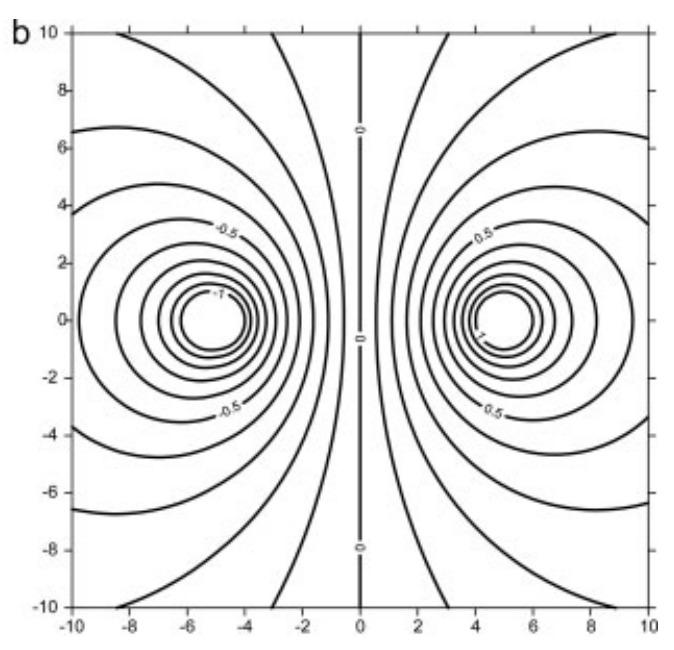

$$
\text { 更 }
$$

FIG. 10. (a) Two parallel cylinder held positive and negative potentials in an infinite plane, (b) contour plot for the present method (42 collocation points), (c) contour plot for the exact solution [32].

\section{Velocity Potential in a Uniform Flow of an Ideal Fluid}

Case 1. Uniform flows past a circular cylinder. Panton [42] has solved the ideal flow about a circular cylinder as shown in Fig. 12(a) with a uniform stream by superposition of a doublet and a stream. The velocity components are computed by complex variables in the cylindrical coordinates as

$$
\begin{aligned}
& v_{r}=v^{\infty}\left(1-\frac{a^{2}}{r^{2}}\right) \cos \theta, \\
& v_{\theta}=-v^{\infty}\left(1+\frac{a^{2}}{r^{2}}\right) \sin \theta,
\end{aligned}
$$

where $v^{\infty}$ is the velocity of the flow far from the cylinders and $a$ is the radius of the cylinder. We also revisited the single-cylinder problem by using the proposed formulation. Our results are 

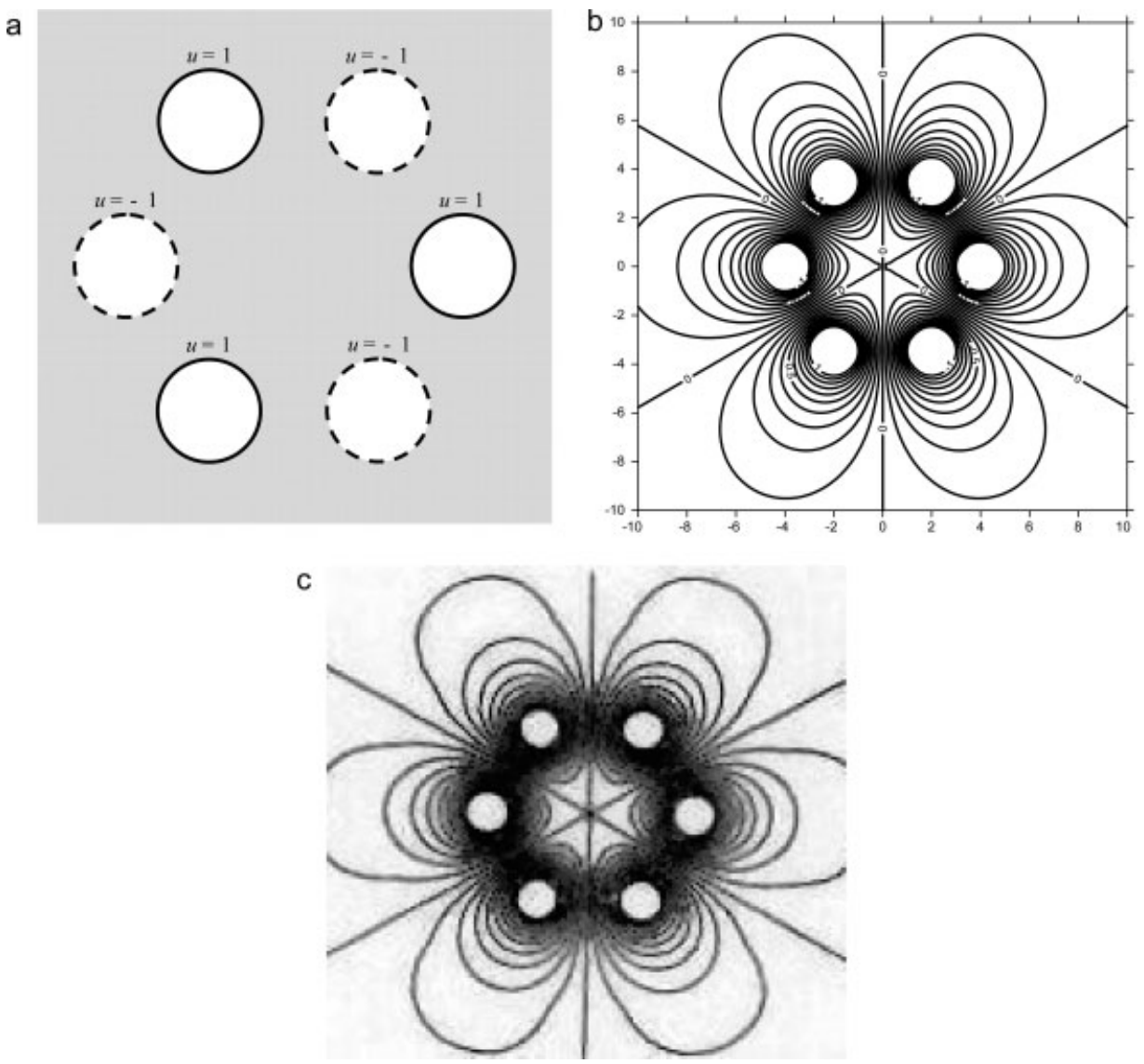

FIG. 11. (a) Hexagonal electrostatic potential in an infinite plane, (b) contour plot for the present method (126 collocation points), (c) contour plot for Onishi's data [44].

consistent with those derived by Panton. The velocity fields with different incident angles $\gamma$ are shown in Fig. 12(b, c).

Case 2. Uniform flows past two parallel cylinders. The other example is referred to [22]. Two parallel cylinders of radius $a$ as shown in Fig. 13(a) with axes a distance $2 l$ apart are placed in a plane-parallel flow of an ideal fluid, making angle $\gamma$ with the line joining the centers of the cylinders. Find the resulting velocity potential. The analytical solution was derived in the bipolar coordinates by Lebedev et al.

$$
\begin{aligned}
u(\alpha, \beta)= & v^{\infty} \sqrt{l^{2}-a^{2}} \\
& \times\left\{\cos \gamma\left[\frac{\sin h(\alpha)}{\cos h(\alpha)+\cos \beta}+2 \sum_{n=1}^{\infty}(-1)^{n} \frac{e^{-n \alpha_{0}}}{\cos h\left(n \alpha_{0}\right)} \sin h(n \alpha) \cos n \beta\right]\right. \\
& \left.+\sin \gamma\left[\frac{\sin \beta}{\cos h(\alpha)+\cos \beta}+2 \sum_{n=1}^{\infty}(-1)^{n} \frac{e^{-n \alpha_{0}}}{\sin h\left(n \alpha_{0}\right)} \cos h(n \alpha) \sin n \beta\right]\right\},
\end{aligned}
$$


a

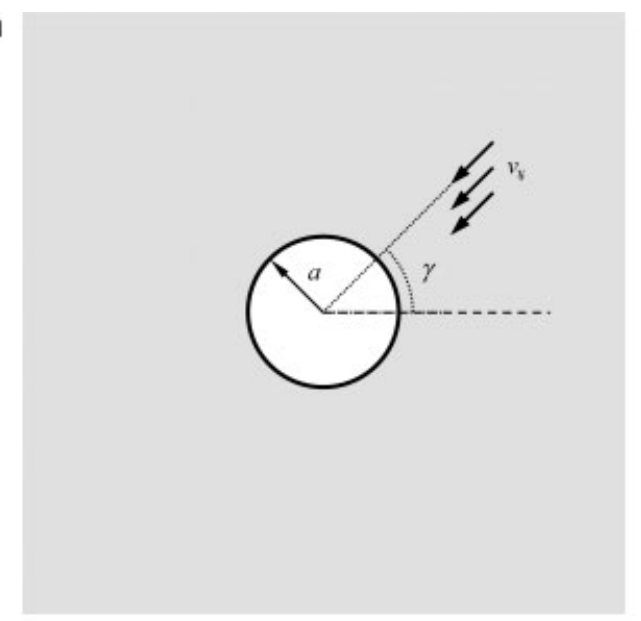

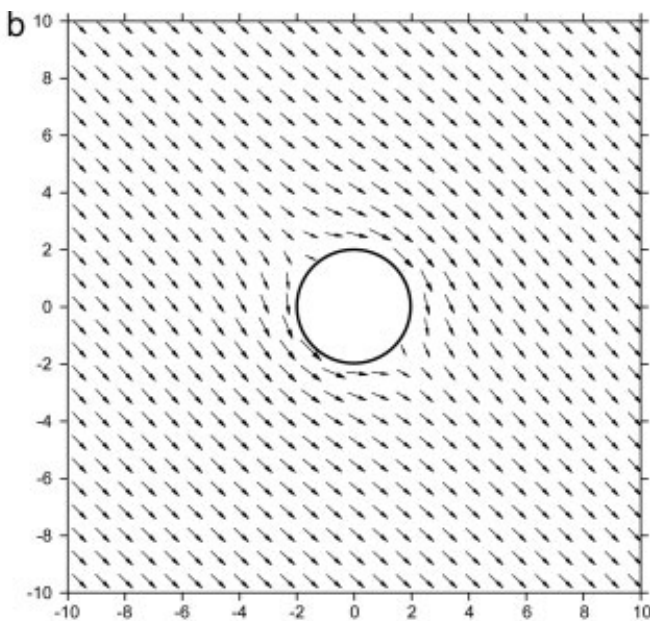

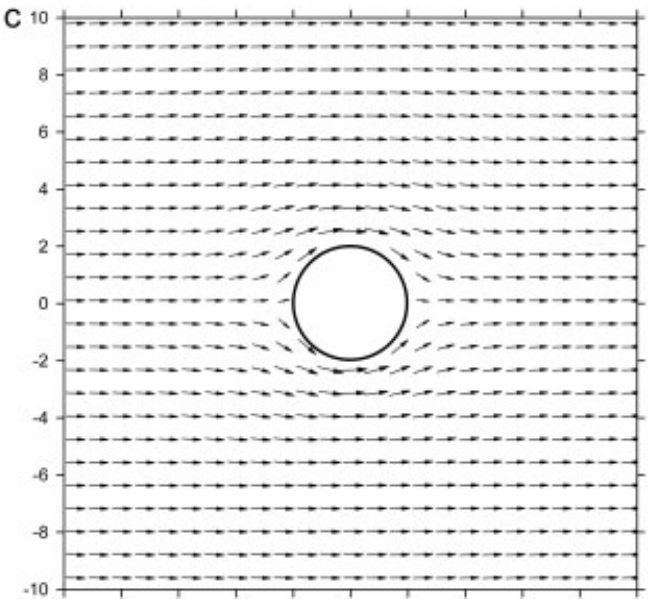

FIG. 12. (a) Flow of an ideal fluid past single cylinder [27], (b) velocity field ( $\left.\gamma=\frac{3 \pi}{4}\right)$, (c) velocity field $(\gamma=\pi)$.

where $\cos h\left(\alpha_{0}\right)=l / a$ and $v^{\infty}$ is the velocity of the flow far from the cylinders. The velocity fields are plotted as shown in Fig. 13(b, c) when the angle $\gamma$ is $3 \pi / 4$ and $\pi$. For such unbounded problems, FEM is not user friendly and needs truncation of the domain.

\section{Applications to Half-Plane Problems}

Half-plane problems with circular holes [22, 43-47] are considered as the special cases. For the special cases, the image method $[19,23]$ was employed to satisfy the boundary condition. To avoid the boundary integral along $B_{0}$ in Eq. (9) and to satisfy the homogeneous Dirichlet boundary condition, the Green's function using the image point is obtained

$$
U\left(\mathrm{~s} ; \mathrm{x}, \mathrm{x}^{\prime}\right)=\ln |\mathrm{x}-\mathrm{s}|-\ln \left|\mathrm{x}^{\prime}-\mathrm{s}\right|,
$$


a

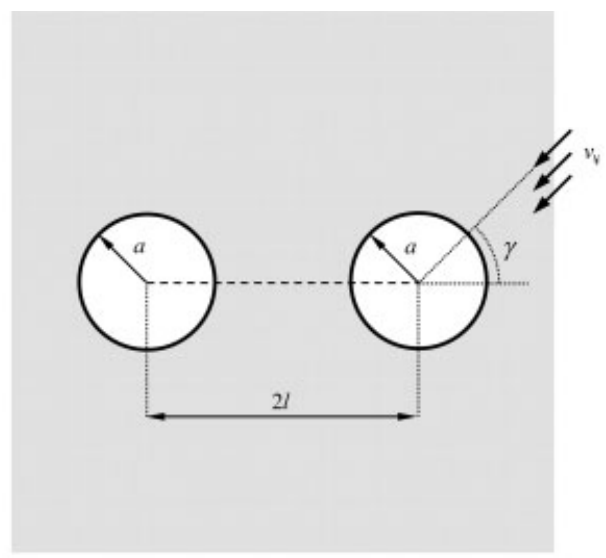

b

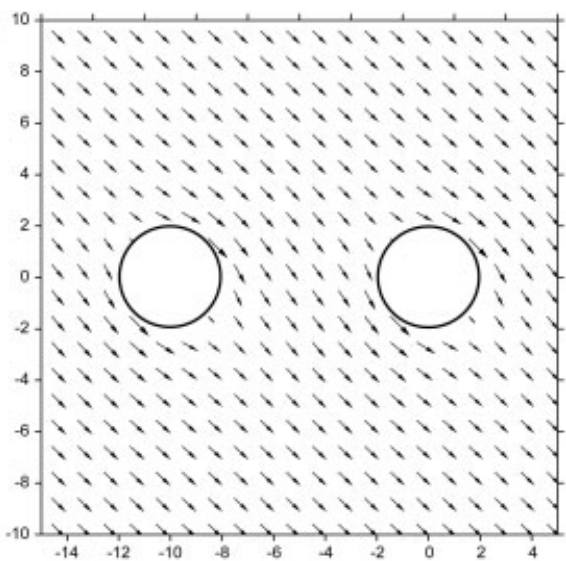

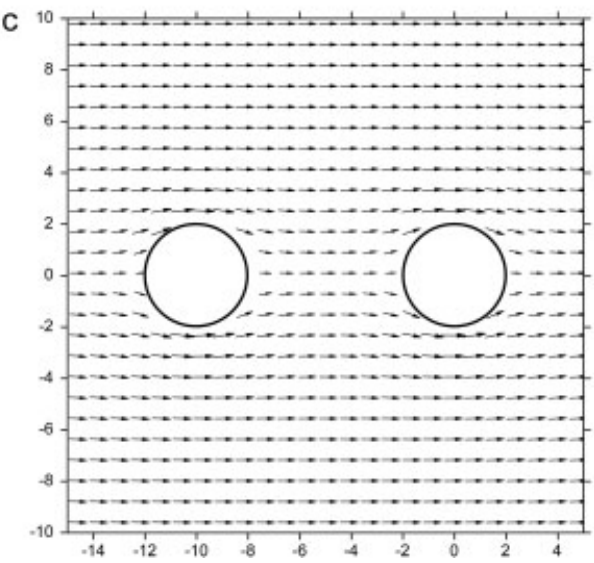

FIG. 13. (a) Flow of an ideal fluid past two parallel cylinders [32], (b) velocity field $\left(\gamma=\frac{3 \pi}{4}\right)$, (c) velocity field $(\gamma=\pi)$.

such that

$$
\left.U\left(\mathrm{~s} ; \mathrm{x}, \mathrm{x}^{\prime}\right)\right|_{\mathrm{s} \in B_{0}}=0 .
$$

Then, Eqs. (3) and (9) are reduced to

$$
\begin{gathered}
2 \pi u(\mathrm{x})=\int_{B} T\left(\mathrm{~s} ; \mathrm{x}, \mathrm{x}^{\prime}\right) u(\mathrm{~s}) d B(\mathrm{~s})-\int_{B} U\left(\mathrm{~s} ; \mathrm{x}, \mathrm{x}^{\prime}\right) t(\mathrm{~s}) d B(\mathrm{~s}), \mathrm{x} \in D, \\
0=\int_{B} T\left(\mathrm{~s} ; \mathrm{x}, \mathrm{x}^{\prime}\right) u(\mathrm{~s}) d B(\mathrm{~s})-\int_{B} U\left(\mathrm{~s} ; \mathrm{x}, \mathrm{x}^{\prime}\right) t(\mathrm{~s}) d B(\mathrm{~s}), \mathrm{x} \in D^{c},
\end{gathered}
$$

where $U\left(\mathrm{~s} ; \mathrm{x}, \mathrm{x}^{\prime}\right)$ denotes the Green's function. On the basis of the Fig. 14, we employ the image concept and obtain

$$
U\left(\mathrm{~s}, \mathrm{x}^{\prime}\right)=\ln r^{\prime}=\left\{\begin{array}{c}
U^{i}\left(R, \theta ; \rho^{\prime}, \phi^{\prime}\right)=\ln R-\sum_{m=1}^{\infty} \frac{1}{m}\left(\frac{\rho^{\prime}}{R}\right)^{m} \cos m\left(\theta-\phi^{\prime}\right), R>\rho^{\prime} \\
U^{e}\left(R, \theta ; \rho^{\prime}, \phi^{\prime}\right)=\ln \rho^{\prime}-\sum_{m=1}^{\infty} \frac{1}{m}\left(\frac{R}{\rho^{\prime}}\right)^{m} \cos m\left(\theta-\phi^{\prime}\right), \rho^{\prime}>R
\end{array},\right.
$$




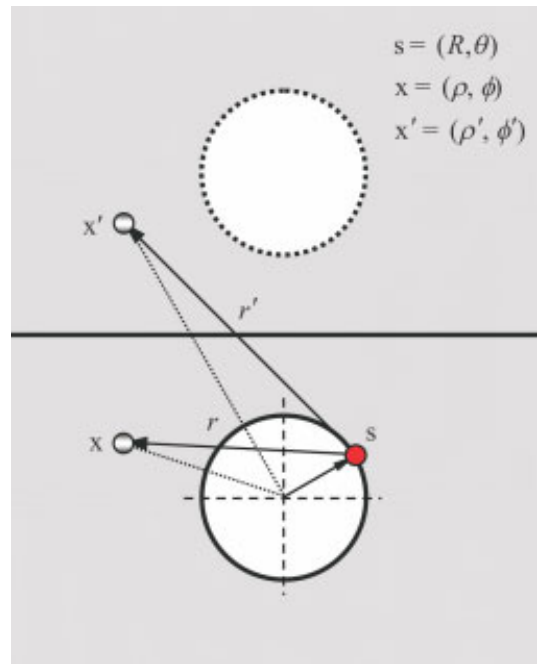

FIG. 14. Considered problem and the auxiliary system. [Color figure can be viewed in the online issue, which is available at www.interscience.wiley.com.]

where $\mathrm{x}^{\prime}=\left(\rho^{\prime}, \phi^{\prime}\right)$ is the image point outside the half-plane domain. The degenerate kernels for the Green's function are

$$
\begin{aligned}
U\left(\mathrm{~s} ; \mathrm{x}, \mathrm{x}^{\prime}\right)= & \ln r-\ln r^{\prime} \\
= & \begin{aligned}
U^{i}\left(R, \theta ; \rho, \phi, \rho^{\prime}, \phi^{\prime}\right)= & \ln R-\sum_{m=1}^{\infty} \frac{1}{m}\left(\frac{\rho}{R}\right)^{m} \cos m(\theta-\phi) \\
& -\ln \rho^{\prime}+\sum_{m=1}^{\infty} \frac{1}{m}\left(\frac{R}{\rho^{\prime}}\right)^{m} \cos m\left(\theta-\phi^{\prime}\right), \rho^{\prime}>R \geq \rho \\
U^{e}\left(R, \theta ; \rho, \phi, \rho^{\prime}, \phi^{\prime}\right)= & \ln \rho-\sum_{m=1}^{\infty} \frac{1}{m}\left(\frac{R}{\rho}\right)^{m} \cos m(\theta-\phi) \\
& -\ln \rho^{\prime}+\sum_{m=1}^{\infty} \frac{1}{m}\left(\frac{R}{\rho^{\prime}}\right)^{m} \cos m\left(\theta-\phi^{\prime}\right), \rho^{\prime}>\rho>R
\end{aligned},
\end{aligned}
$$

After taking the normal derivative with respect to Eq. (38), the kernel function, $T\left(\mathrm{~s} ; \mathrm{x}, \mathrm{x}^{\prime}\right)$, can be derived as

$$
T\left(\mathrm{~s} ; x, \mathrm{x}^{\prime}\right)=\left\{\begin{array}{rl}
T^{i}\left(R, \theta ; \rho, \phi, \rho^{\prime}, \phi^{\prime}\right)= & -\frac{1}{R}-\sum_{m=1}^{\infty}\left(\frac{\rho^{m}}{R^{m+1}}\right) \cos m(\theta-\phi) \\
& -\sum_{m=1}^{\infty}\left(\frac{R^{m-1}}{\rho^{\prime m}}\right) \cos m\left(\theta-\phi^{\prime}\right), \rho^{\prime}>R>\rho \\
T^{e}\left(R, \theta ; \rho, \phi, \rho^{\prime}, \phi^{\prime}\right)= & \sum_{m=1}^{\infty}\left(\frac{R^{m-1}}{\rho^{m}}\right) \cos m(\theta-\phi) \\
& -\sum_{m=1}^{\infty}\left(\frac{R^{m-1}}{\rho^{\prime m}}\right) \cos m\left(\theta-\phi^{\prime}\right), \rho^{\prime}>\rho>R
\end{array} .\right.
$$

First, a steady state heat transfer problem with semicircle removed [46] is considered as shown in Fig. 15(a). After introducing the image concept, we determine the isotherms in the semi-infinite 
a

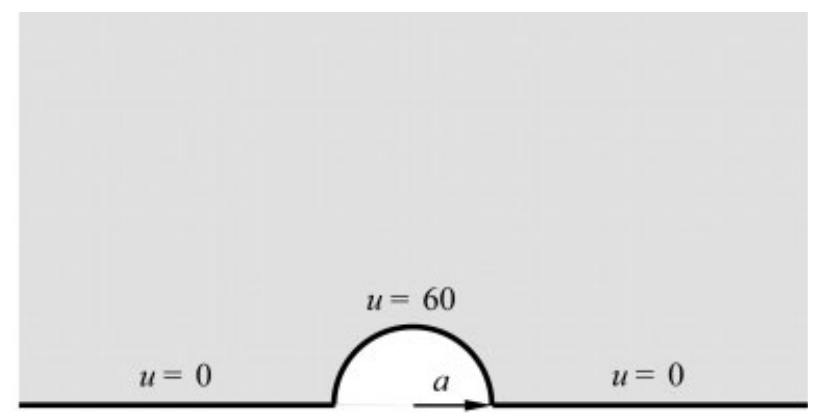

b

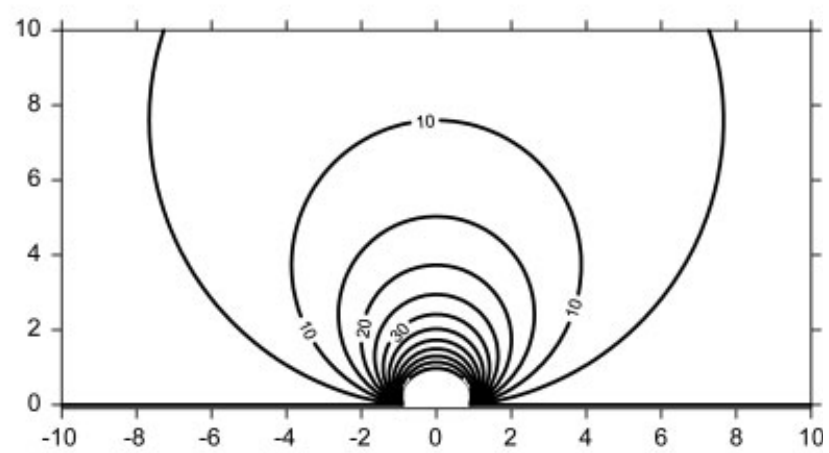

C

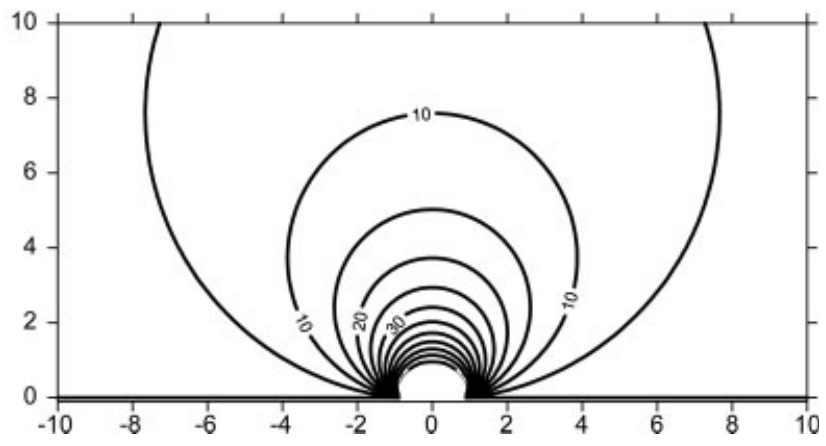

FIG. 15. (a) Steady state heat conduction in a half-plane with semicircle removed, (b) contour plot for the present method (21 collocation points), (c) contour plot for the analytical solution [42].

plane. Figure 15(b, c) show the contour plot for the present method and the analytical solution, respectively. Figure 16(a) give the sketches of the half-plane problems with mixed-type boundary conditions. Figure 16(b) denotes the contour plot of potential using the present method, and Fig. 16(c) is the available exact solution [22]. The relative error is addressed in Fig. 16(d), and it is found that the results of mixed-type case agree well after comparing them with the exact solution.

\section{CONCLUDING REMARKS}

For the Laplace problems with circular boundaries, we have proposed a special BIEM by using degenerate kernels, null-field integral equation and Fourier series in an adaptive observer system. 

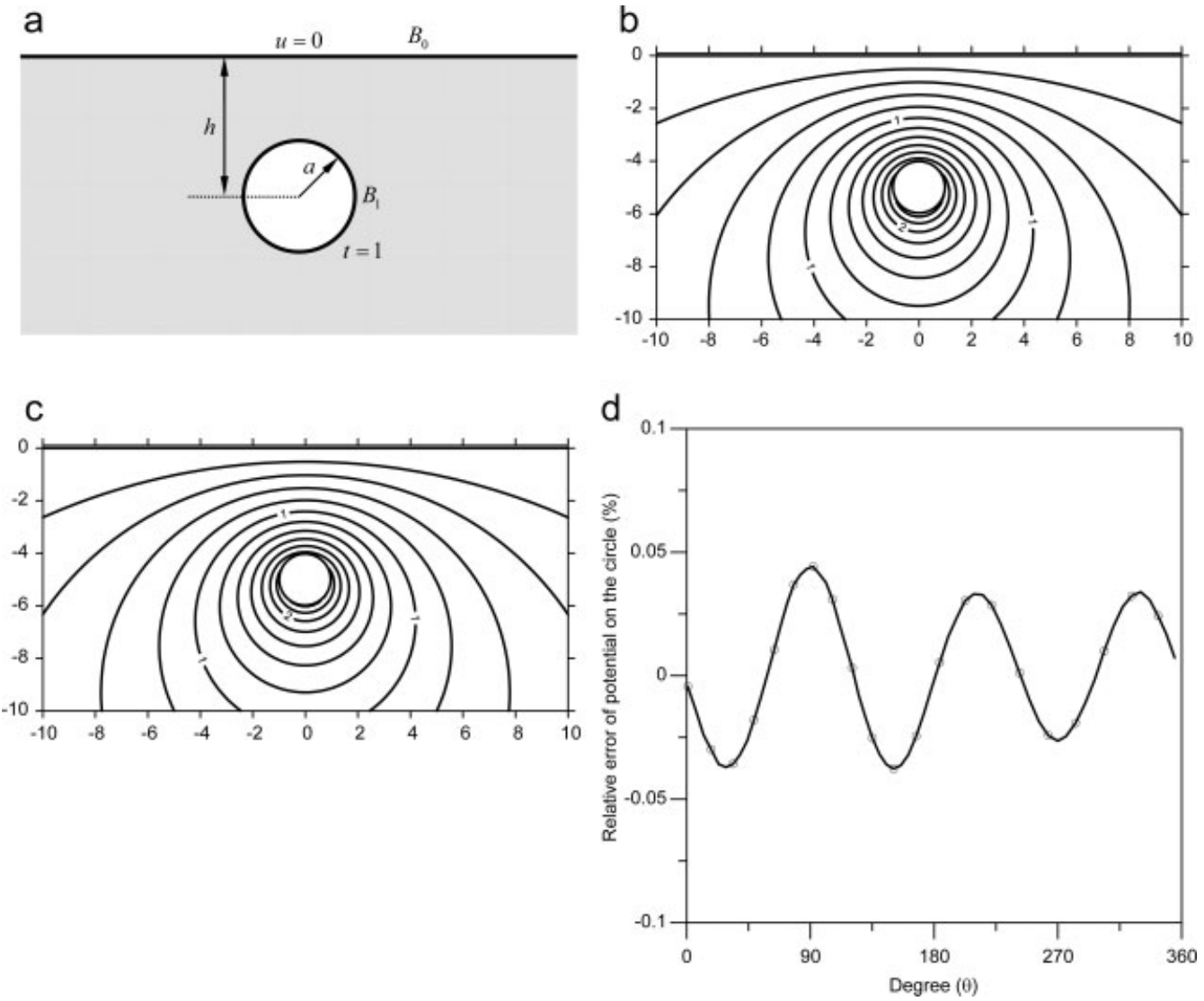

FIG. 16. (a) Half-plane problem subject to mixed-type boundary conditions, (b) contour plot for the present method (21 collocation points), (c) contour plot for the exact solution [32], (d) relative error of boundary potential by using the present method.

The method shows great generality and versatility for the problems with multiple circular holes of arbitrary radii and positions. Numerical results agree very well with the available exact solution and FEM (ABAQUS) data for only ten terms in Fourier series. Engineering problems with circular boundary which satisfy the Laplace equation can be solved by using the proposed approach in the unified manner.

\section{References}

1. M. J. Balcerzak and S. Raynor, Steady state temperature distribution and heat flow in prismatic bars with isothermal boundary conditions, Int J Heat and Mass Transfer 3 (1961), 113-125.

2. M. R. Barone and D. A. Caulk, Optimal arrangement of holes in a two-dimensional heat conductor by a special boundary integral method, Int J Numer Methods Eng 18 (1982), 675-685.

3. M. D. Bird and C. R. Steele, A solution procedure for Laplace's equation on multiply-connected circular domains, ASME J Appl Mech 59 (1992), 398-404.

4. D. A. Caulk, Analysis of steady heat conduction in regions with circular holes by a special boundary integral method, IMA J Appl Math 30 (1983), 231-246. 
5. D. A. Caulk, Steady heat conduction from an infinite row of holes in a half-space or a uniform slab, Int J Heat Mass Transfer 26 (1983), 1509-1513.

6. K. D. Cole, Fast-converging series for heat conduction in the circular cylinder, J Eng Math 49 (2004), 217-232.

7. H. W. Cheng and L. Greengard, On the numerical evaluation of electrostatic field in dense random dispersions of cylinders, J Comput Phys 136 (1997), 629-639.

8. J. W. Craggs, The determination of capacity for two-dimensional system of cylindrical conductors, Q J Math 17 (1946), 131-137.

9. M. A. Jaswon and G. T. Symm, Integral equation methods in potential theory and electrostatics, Academic Press, New York, 1977.

10. D. Palaniappan, Electrostatics of two intersecting conducting cylinders, Math Comput Modelling 36 (2002), 821-830.

11. H. W. Richmond, On the electrostatic field of a plane or circular grating formed of thick rounded bars, Proc London Math Soc 22 (1923), 389-403.

12. R. Barnes and I. Jankovic, Two-dimensional flow through large numbers of circular inhomogeneities, J Hydrol 226 (1999), 204-210.

13. I. Jankovic and R. Barnes, Three-dimensional flow through large numbers of spheroidal inhomogeneities, J Hydrol 226 (1999), 224-233.

14. D. K. Lee, Image singularity system to represent two circular cylinders of different diameter, ASME J Fluids Eng 122 (2000), 715-719.

15. W. T. Ang and I. Kang, A complex variable boundary element method for elliptic partial differential equations in a multiply-connected region, Int J Comput Math 75 (2000), 515-525.

16. S. I. Chou, Stress field around holes in antiplane shear using complex variable boundary element method, ASME J Appl Mech 64 (1997), 432-435.

17. E. Honein, T. Honein, and G. Herrmann, Further aspects of the elastic field for two circular inclusions in antiplane elastostatics, ASME J Appl Mech 59 (1992), 774-779.

18. E. Honein, T. Honein, and G. Herrmann, On two circular inclusions in harmonic problem, Q Appl Math 50 (1992), 479-499.

19. E. Honein, T. Honein, and G. Herrmann, Energetics of two circular inclusions in anti-plane elastostatics, Int J Solids Struct 37 (2000), 3667-3679.

20. D. A. Caulk, Analysis of elastic torsion in a bar with circular holes by a special boundary integral method, ASME J Appl Mech 50 (1983), 101-108.

21. C. B. Ling, Torsion of a circular tube with longitudinal circular holes, Q Appl Math 5 (1947), 168-181.

22. N. N. Lebedev, I. P. Skalskaya, and Y. S. Uyand, Worked problem in applied mathematics, Dover Publications, New York, 1979.

23. S. X. Gong and S. A. Meguid, Interacting circular inhomogeneities in plane elastostatics, Acta Mech 99 (1993), 49-60.

24. S. X. Gong, Antiplane interaction among multiple circular inclusions, Mech Res Commun 22 (1995), 257-262.

25. J. T. Chen, H.-K. Hong, I. L. Chen, and K. H. Chen, Nonuniqueness and its treatment in the boundary integral equations and boundary element methods, CMC2003 Plenary lecture, Hsin-Chu (2003).

26. S. L. Crouch and S. G. Mogilevskaya, On the use of Somigliana's formula and Fourier series for elasticity problems with circular boundaries, Int J Numer Methods in Eng 58 (2003), 537-578.

27. S. G. Mogilevskaya and S. L. Crouch, A Galerkin boundary integral method for multiple circular elastic inclusions, Int J Numer Methods Eng 52 (2001), 1069-1106. 
28. M. R. Barone and D. A. Caulk, Special boundary integral equations for approximate solution of Laplace's equation in two-dimensional regions with circular holes, Q J Mech and Appl Math 34 (1981), 265-286.

29. M. R. Barone and D. A. Caulk, Special boundary integral equations for approximate solution of potential problems in three-dimensional regions with slender cavities of circular cross-section, IMA J Appl Math 35 (1985), 311-325.

30. M. R. Barone and D. A. Caulk, Analysis of liquid metal flow in die casting, Int J Eng Sci 38 (2000), 1279-1302.

31. M. D. Bird and C. R. Steele, Separated solution procedure for bending of circular plates with circular holes, ASME Appl Mech Rev 44 (1991), 27-35.

32. R. Kress, Linear integral equations, Springer-Verlag, Berlin, 1989.

33. J. T. Chen, C. C. Hsiao, and S. Y. Leu, A new method for Stokes' flow with circular boundaries using degenerate kernel and Fourier series, Int J Numer Methods Eng, in press.

34. J. T. Chen and H.-K. Hong, Review of dual boundary element methods with emphasis on hypersingular integrals and divergent series, ASME Appl Mech Rev 52 (1999), 17-33.

35. J. T. Chen, W. C. Shen, and P. Y. Chen, Analysis of circular torsion bar with circular holes using null-field approach, Comput Modelling Eng Sci 12 (2006), 109-119.

36. J. T. Chen and Y. P. Chiu, On the pseudo-differential operators in the dual boundary integral equations using degenerate kernels and circulants, Eng Anal Boundary Elem 26 (2002), 41-53.

37. J. T. Chen, J. H. Lin, S. R. Kuo, and Y. P. Chiu, Analytical study and numerical experiments for degenerate scale problems in boundary element method using degenerate kernels and circulants, Eng Anal Boundary Elem 25 (2001), 819-828.

38. J. T. Chen, S. R. Kuo, and J. H. Lin, Analytical study and numerical experiments for degenerate scale problems in the boundary element method for two-dimensional elasticity, Int J Numer Methods Eng 54 (2002), 1669-1681.

39. J. T. Chen, C. F. Lee, I. L. Chen, and J. H. Lin, An alternative method for degenerate scale problem in boundary element methods for the two-dimensional Laplace equation, Eng Anal Boundary Elem 26 (2002), 559-569.

40. G. F. Carrier and C. E. Pearson, Partial differential equations, Academic Press, New York, 1976.

41. K. Onishi, Numerical methods for PDE, Tokyo University Press, Tokyo, 1991.

42. R. L. Panton, Incompressible flow, Wiley-Interscience, New York, 1984.

43. C. B. Kooi and A. Verruijt, Interaction of circular holes in an infinite elastic medium, Tunneling Underground Space Technol 16 (2001), 59-62.

44. A. Verruijt, A complex variable solution for a deforming circular tunnel in an elastic half-plane, Int $\mathbf{J}$ Numer Anal Methods Geomech 21 (1997), 77-89.

45. A. Verruijt, Deformations of an elastic half plane with a circular cavity, Int J Solids Struct 35 (1998), 2795-2804.

46. M. R. Spiegel, Theory and problems of complex variables with an introduction to conformal mapping and its application, McGraw-Hill, New York, 1981.

47. M. D. Greenberg, Applications of green's function in science and engineering, Prentice-Hall, New Jersey, 1971. 